Please do not remove this page

RMIT

UNIVERSITY

\title{
Investigating the potential for using a simple water reaction turbine for power production from low head hydro resources
}

Date, Abhijit Shridhar; Date, Ashwin Shridhar; Akbarzadeh, Aliakbar

https://researchrepository.rmit.edu.au/esploro/outputs/9921858635201341/filesAndLinks?institution=61RMIT_INST\&index=null

Date, A. S., Date, A. S., \& Akbarzadeh, A. (2013). Investigating the potential for using a simple water reaction turbine for power production from low head hydro resources. Energy Conversion and Management, 66, 257-270. https://doi.org/10.1016/j.enconman.2012.09.032

Published Version: https://doi.org/10.1016/j.enconman.2012.09.032

Repository homepage: https://researchrepository.rmit.edu.au

(C) 2012 Elsevier

Downloaded On 2023/04/27 01:25:02 +1000

Please do not remove this page 
Thank you for downloading this document from the RMIT Research Repository.

The RMIT Research Repository is an open access database showcasing the research outputs of RMIT University researchers.

RMIT Research Repository: http://researchbank.rmit.edu.au/

\section{Citation:}

Date, A, Date, A and Akbarzadeh, A 2013, 'Investigating the potential for using a simple water reaction turbine for power production from low head hydro resources', Energy Conversion and Management, vol. 66, pp. 257-270.

See this record in the RMIT Research Repository at:

http://researchbank.rmit.edu.au/view/rmit:17983

Version: Accepted Manuscript

Copyright Statement: () 2012 Elsevier

Link to Published Version:

http://dx.doi.org/10.1016/j.enconman.2012.09.032 


\title{
Investigating the potential of using simple reaction water
}

\section{turbine for power production from low head hydro resources}

\author{
Abhijit Date and Aliakbar Akbarzadeh \\ Energy CARE Group, School of Aerospace, Mechanical and Manufacturing Engineering, \\ RMIT University, PO Box 7 1, Bundoora, Victoria 3083, Australia
}

\begin{abstract}
In this analysis the simple reaction water turbine known as Barker's Mill is revisited. The major geometrical and operational parameters have been identified and using principles of conservation of mass, momentum and energy the governing equations have been developed for the ideal case of zero frictional losses. The solutions of the resulting equations are then offered in a non-dimensional form. It is shown that the maximum torque produced by the machine is for the case when the turbine is stationary. At this point the net output power is zero. As the load torque is decreased the turbine starts rotating and power is produced. Furthermore, due to a centrifugal pumping effect, the mass flow rate of water through the turbine increases during acceleration. Further decrease in the load torque is accompanied by increase in speed, output power, water mass flow rate and efficiency. It is shown that when the load torque is reduced towards half the value of the torque at the stationary condition, then water mass flow rate, speed and output power approach infinity. Under this condition the efficiency of the machine approaches unity. The non-dimensional presentation of the characteristics of the idealized turbine is used to investigate the characteristics of the machine and to explore its application for production of power from water reservoirs of low heads. Theoretical
\end{abstract}


analysis of a simple reaction turbine considering the fluid frictional losses for practical situation has been presented. A practical turbine will never runaway reach infinite speed and the maximum power and efficiency of such turbine will only depend on the fluid frictional losses. Here a new factor that represents the overall fluid frictional losses within the turbine has been defined. Finally this paper briefly presents the experimental performance results for two simple reaction water turbine prototypes. The two turbine prototypes under investigation have different rotor diameters $\varnothing 0.24 \mathrm{~m}$ and $\varnothing 0.12 \mathrm{~m}$. The two turbine models were tested for the supply head ranging from $1 \mathrm{~m}$ to $4 \mathrm{~m}$. The simple reaction water turbine can operate under very low hydro-static head with high energy conversion efficiency. This type of turbine exhibits prominent self-pumping ability at high rotational speeds. Under low head to achieve high rotational speeds the turbine diameter should be very small and this limits the volumetric capacity and hence the power generation capacity of such a turbine. So the practical applications of this turbine would be limited to the micro-hydro power generation. The split pipe design of the reaction turbine is easy to manufacture and have proven to have energy conversion efficiency of around 50\% even under low heads.

Keywords: Simple Reaction Turbine; Water Turbine; Low Head Hydro; Hydro Electric.

\section{Nomenclature}

A Total nozzle exit area $\left(\mathrm{m}^{2}\right)$

D Nozzle diameter (m)

$D_{c} \quad$ Nozzle equivalent diameter (m)

$g$ Acceleration due to gravity $\left(\mathrm{m} / \mathrm{s}^{2}\right)$ 
$H \quad$ Water height in reservoir (m)

$H_{c} \quad$ Centrifugal head (m)

$K_{s} \quad$ Specific speed for turbines

$m \quad$ Mass flow rate of water through the turbine $(\mathrm{kg} / \mathrm{s})$

$m_{s} \quad$ Mass flow rate of water through the turbine when it is stationary $(\mathrm{kg} / \mathrm{s})$.

${ }^{*} m \quad$ Non-dimensional mass flow rate

$\eta \quad$ Efficiency of conversion of potential energy to work

$P_{C} \quad$ Centrifugal pressure $\left(\mathrm{N} / \mathrm{m}^{2}\right)$

Q Volume flow rate $\left(\mathrm{m}^{3} / \mathrm{s}\right)$

$\rho \quad$ Density of water $\left(\mathrm{kg} / \mathrm{m}^{3}\right)$

$R \quad$ Radius of the rotor (m)

$T \quad$ Torque (N-m)

$T_{s} \quad$ Torque when the turbine is stationary $(\mathrm{N}-\mathrm{m})$

${ }^{*} T \quad$ Non-dimensional Torque

$\dot{W} \quad$ Output power (W)

${ }^{*} \dot{W} \quad$ Non-dimensional power

$\omega \quad$ Angular velocity of the rotor ( $\mathrm{rad} / \mathrm{s})$

${ }^{*} \omega \quad$ Non-dimensional angular velocity 
$U \quad$ Tangential velocity of the nozzles $(\mathrm{m} / \mathrm{s})$

${ }^{*} U \quad$ Non-dimensional tangential velocity of the nozzles

$V_{a} \quad$ Absolute velocity of water leaving the nozzle with respect to a stationary observer $(\mathrm{m} / \mathrm{s})$

${ }^{*} V_{a} \quad$ Non-dimensional absolute velocity

$V_{r} \quad$ Relative velocity of water with respect to the nozzle $(\mathrm{m} / \mathrm{s})$

${ }^{*} V_{r} \quad$ Non-dimensional relative velocity

\section{Introduction}

The use of moving water to drive machinery has a long history beginning with undershot and overshot water wheels and progressing through turbines of various designs. Wilson [1] provides an interesting brief history of water turbines to which the reader is referred. Turbines are broadly classified into two types, impulse and reaction, although designs may involve a mixture of the two types of action. In a pure impulse hydraulic turbine, such as the Pelton wheel the energy associated with the pressure in the supply pipe is converted to kinetic energy in the stationary nozzle (or vanes) and the high velocity stream is directed onto moving buckets (or blades). There is no further change in pressure of the water as it moves through the buckets and hence no change in the magnitude of the velocity of the stream relative to the buckets. The buckets however change the direction of the flow, and the movement of the buckets changes the magnitude of the stream's absolute velocity. From the resulting change in momentum of the stream, the impulsive force on the buckets and the torque on the wheel can be calculated. It is desirable, for 
maximum energy extraction from the water that the wheel speed and bucket shape be such that the water leaves the turbine with negligible kinetic energy[2, 3].

In a pure reaction hydraulic turbine the water is still pressurized as it enters the moving parts and as it passes through the moving nozzles or vanes its pressure is reduced and the velocity of the stream relative to the moving parts is increased. From the associated change in absolute velocity and momentum, the reactive force and torque on the turbine nozzle can be calculated. (Note that in the nozzle of the Pelton wheel a reaction force is also generated, but since the nozzle is stationary no mechanical energy is extracted at that point.) The earliest recorded reaction turbine, that of Hero (about 2,000 years ago) [4] was a reaction machine and is illustrated in Fig. 1, some experiments of the modem day Hero type turbines are described by Hsu and Leo [5]and Brady [6]. Barker's mill, which is shown diagrammatically in Fig. 2, was the first hydraulic reaction turbine and was invented in about 1740, this machine was further refined by Pupil in 1775 and Whitelaw in 1839 [1]. One such refinement is to feed the water into the underside of the rotor. By feeding water into the turbine from underneath as shown in Fig. 3 the upward action of the static pressure of the entering feed water may be used to counteract the downward gravitational force on the moving parts thereby reducing the thrust load on bearings supporting these moving parts. 

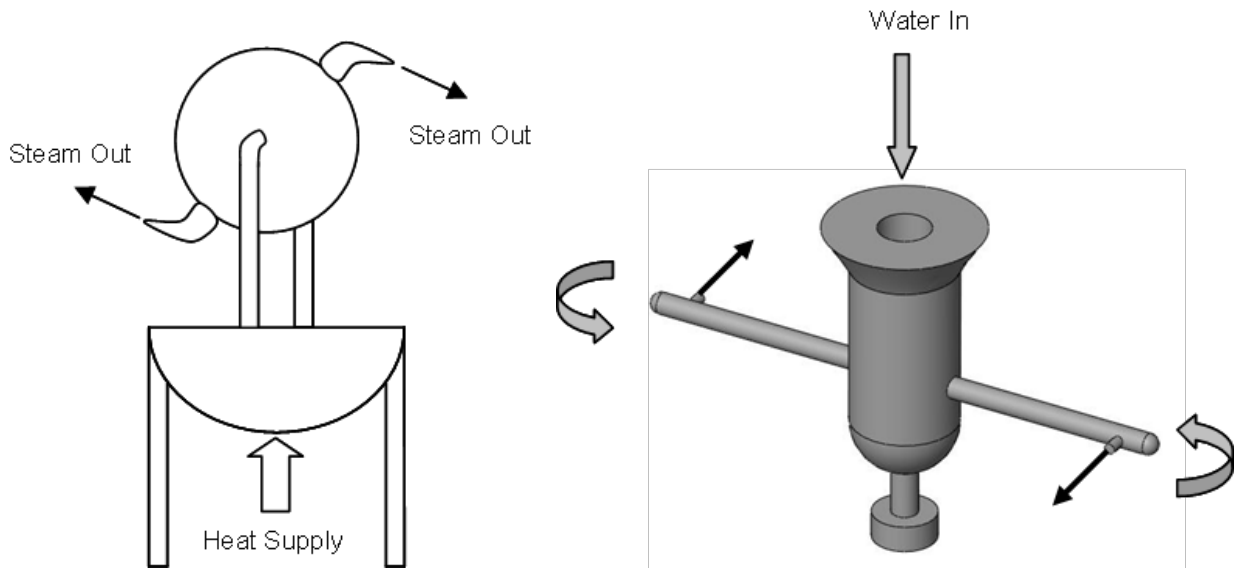

Figure 1 Hero's turbine

Figure 2 Barker's Mill

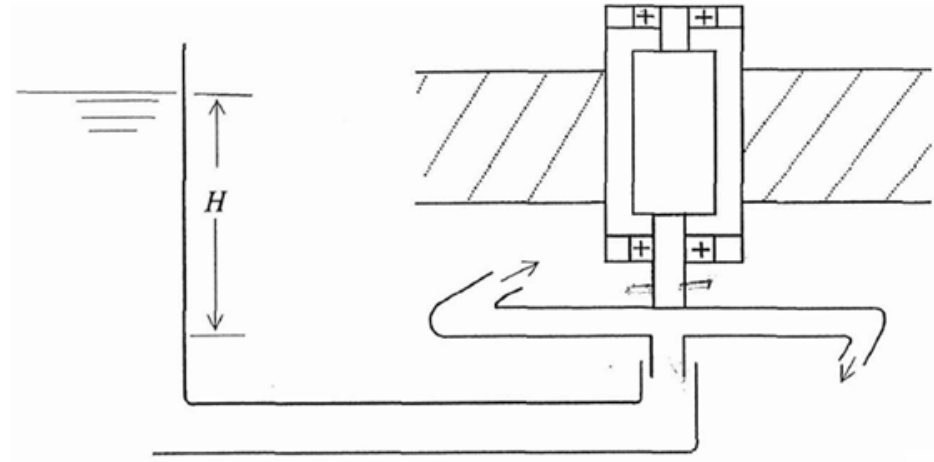

Figure 3 Schematic presentation of feeding water into the turbine from underneath

Simple reaction turbines of the Barker's Mill type have been analyzed in several textbooks such as: Shepherd [4]; Daugherty \& Ingersoll [7]; Balje [8]; and Duncan, Thorn and Young [9] , the most comprehensive being the later. Most analyses observe that such machines are inefficient at low speeds since in that case the water still has significant kinetic energy when it leaves the turbines. They also usually note the centrifugally induced increase in pressure that occurs within the arms as they rotate and the consequent increase in flow rate that occurs. It is the objective of this paper to explore 
in more detail the changes in efficiency, flow rate, torque and power that occur with increase in speed of rotation of an ideal frictionless Barker's Mill.

Through parametric analysis, attempts are also made to undertake a comprehensive study on the characteristics of the frictionless simple reaction water turbine and present its inherent potential as a candidate for application to low head water reservoirs for the production of power. As part of the discussions presented in this work, what the authors believe to be incorrect conclusions, which have been drawn in the published literature, will be pointed out. The conclusions relate to the performance of simple reaction water turbines as their speed tends towards infinity.

In short, it appears that simple reaction water turbines are to some extent misunderstood, under-utilized and almost forgotten other than for garden sprinklers. The aim of this paper is to demonstrate the main features of these devices and to propose potential application to renewable electrical power generation.

\section{Theoretical analysis of idealized simple reaction turbine}

In this section attempts are made to provide governing equations for prediction of the performance of a simple reaction water turbine. These equations are then used for parametric study of the performance of the machine under specified conditions.

Let us assume that total head of $\mathrm{H}(\mathrm{m})$ is available in a water reservoir and the aim is to convert the potential energy of water to useful work by the means of a simple reaction turbine as shown in Fig 3.

Here we neglect all losses due to friction such as losses due to the flow of water from the reservoir, and through the piping, the rotor and the nozzles. We also neglect all the other 
mechanical losses such as windage losses due to the rotation of the rotor as well as the frictional losses of the bearings. The water is assumed to be incompressible. Considering the schematic shown in Fig. 4 we can write:

$$
U=R \omega
$$

And

$$
V_{a}=V_{r}-U
$$

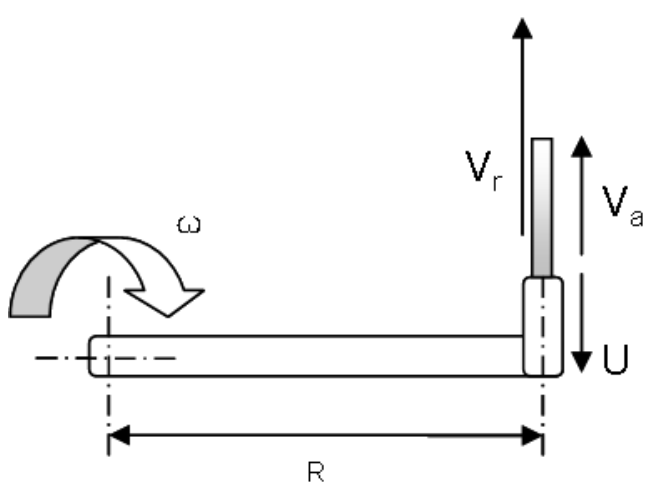

Figure 4 Schematic showing the rotor, the nozzle and the velocities

In case of a simple reaction turbine there are two components of pressures acting that govern the flow of water through the turbine. The main operating head is created due to physical difference between the water level in the reservoir and the position of the turbine, whereas the secondary head is created due to angular speed of the turbine. Considering a position just upstream of the nozzle (where the flow cross sectional area is much greater than the nozzle exit area $A$ there are two components of pressure: that due to the head in the reservoir $(\rho g H)$ and that due to the centrifugal pumping 
action $\left(P_{c}=1 / 2 \rho \omega^{2} R^{2}\right)$. The combined effect of these pressures is to drive the water out at a velocity relative to the nozzle found from:

$$
1 / 2 \rho V_{r}^{2}=\rho g H+1 / 2 \rho \omega^{2} R^{2}
$$

Alternatively it can be rewritten as:

$$
1 / 2 \rho V_{r}^{2}=\rho g\left(H+H_{c}\right)
$$

Where the centrifugal head,

$$
H_{c}=\frac{\omega^{2} R^{2}}{2 g}
$$

From Eq.(3):

$$
V_{r}=\sqrt{2 g H+\omega^{2} R^{2}}
$$

Mass flow out of the nozzle may be found from:

$$
\dot{m}=\rho V_{r} A
$$

Where,

$$
\dot{m}=\rho A\left(\sqrt{2 g H+\omega^{2} R^{2}}\right)
$$

By consideration of Eq. (8) it can be seen that the mass flow is at a minimum when the turbine is stationary and increases as the turbine angular velocity increases. This self 
pumping effect is mentioned qualitatively by J.R. Ainsworth Davis [10]. This centrifugal head or self-pumping phenomenon is similar to working principle of a centrifugal pump. The minimum value which occurs when the turbine is stationary we have called,

$$
\dot{m}_{s}=\rho A \sqrt{2 g H}
$$

Fig. 5 shows mass flow rate of water as a function of rotational speed for several different heads applied to a machine for which $A=2 \times 10^{-4} \mathrm{~m}^{2}$ and $R=0.125 \mathrm{~m}$.

Momentum balance results in,

$$
T=\dot{m} V_{a} R
$$

The produced power can be related to torque $T$ and angular velocity $\omega$ in the following form

$$
\dot{W}=T \omega
$$

Using conservation of energy we also obtain, assuming, as stated earlier, no frictional losses:

$$
\dot{m} g H=w+1 / 2 \dot{m} V_{a}^{2}
$$

Where,

$$
\dot{W}=\dot{m} g H+1 / 2 \dot{m} V_{a}^{2}
$$




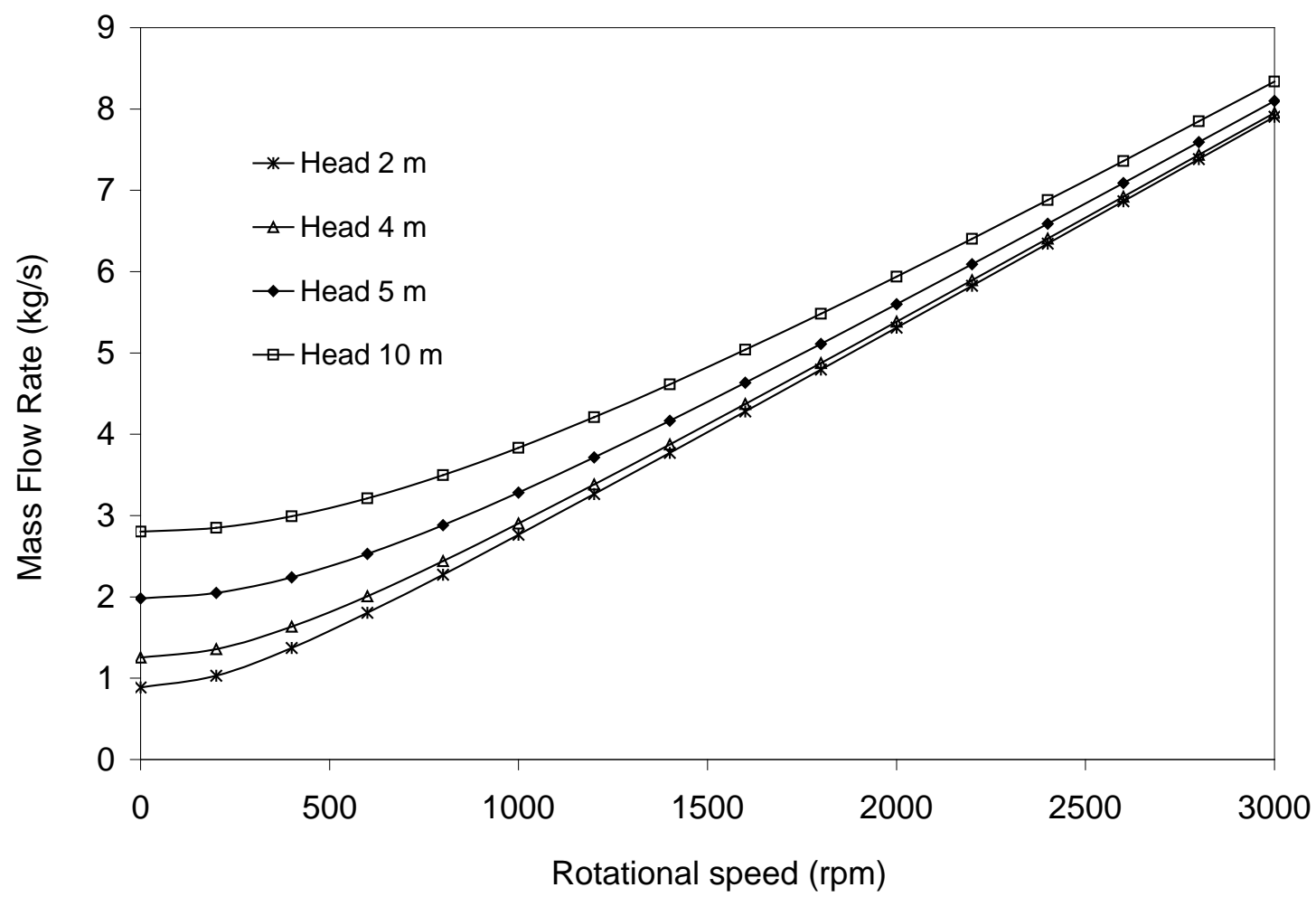

Figure 5 Variation of mass flow rate with rotational speed for several water heads

The efficiency of conversion of potential energy to useful work can be written as,

$$
\eta=\frac{\dot{W}}{\dot{m} g H}
$$

As the speed $\mathrm{U}(R \omega)$ of the machine is increased it can be seen from Eq. 6 that $V_{r}$ approaches $U$ and hence from Eq. 2 the absolute velocity $V_{a}$ approaches zero. By consideration of Eq. 10 and 11 it can be seen that if $V_{a}$ approaches zero the torque and power approach zero also if the mass flow rate is fixed (and such fixed flow rate situations are considered for a simple steam reaction turbine in Hsu and Leo [5]. However as has been shown in Eq. 8 and Fig. 5 the centrifugal action is such as to make the mass flow rate increase with speed. The combined effect on the torque (Eq.10) and 
power (Eq.11) of these two opposing trends of decreasing $V_{a}$ versus increasing $m$ is not immediately apparent, and is explored subsequently in this paper. Duncan [9] state that when $V_{r}=\omega R$ the power is zero, but do not explore the aforementioned combined effect on power of $\dot{m}$ approaching infinity as $V_{r}$ and $\omega R$ approach each other at high speed. Similarly Daugherty [7] associate the runaway high speed condition with zero torque due to the absolute fluid velocity approaching zero without considering the opposing trend of mass flow approaching infinity. Duncan [9] however discuss the tendency towards instability of the outward flow turbine. Instability in this context is the characteristic of having a self enhancing runaway tendency if the torque is reduced. By contrast, inward flowing turbines such as the Thomson and Francis are self governing to some extent in that a speeding up causes a build up of centrifugal pressure that tends to reduce the inward mass flow and hence the torque.

In the above equations $R, A$ and $H$ are known geometrical parameters, $\rho$ is the density of water and $g$ is the acceleration due to gravity. Here the solutions of the above algebraic equations are offered based on the load torque $T$. By solving equations (1) to (14) the equations relating the seven unknowns i.e., $U, V_{a}, V_{r}, \omega, \dot{m}, \dot{W}$ and $\eta$ to the known parameters of the system can be developed.

We have chosen to offer the solutions in a particular non-dimensional form for ease of presentation and generalization of the results. As found in Eq. 9, $\dot{m}_{s}$ is the mass flow rate through the turbine when the turbine is stationary.

This mass flow rate causes a reaction, which needs to be balanced by $T_{s}$ which is given by: 


$$
T_{s}=\dot{m}_{s} V_{a} R=\rho A \sqrt{2 g H} \sqrt{2 g H} R
$$

Therefore:

$T_{s}=2 \rho A R g H$

Now we define the following non-dimensional parameters:

$$
\begin{aligned}
& { }^{*} T=\frac{T}{T_{s}} \\
& { }^{*} \dot{m}=\frac{\dot{m}}{\dot{m}_{s}} \\
& { }^{*} V_{a}=\frac{V_{a}}{\sqrt{2 g H}} \\
& { }^{*} V_{r}=\frac{V_{r}}{\sqrt{2 g H}} \\
& { }^{*} U=\frac{U}{\sqrt{2 g H}} \\
& { }^{*} \omega=\frac{\omega}{\left(\frac{\sqrt{2 g H}}{R}\right)}
\end{aligned}
$$

And, 


$$
{ }^{*} \dot{W}=\frac{\dot{W}}{\dot{m}_{s} g H}
$$

Based on equations (9) \& (16) and using the definitions introduced in equations (17) to (23) the solutions to equations (1), (2), (7), (10), (11), (13), and (14) can be obtained.

Using ${ }^{*} T$ as the independent variable enables explicit expressions to be obtained for the other non-dimensional variables in equations (17) - (23)

$$
\begin{aligned}
& { }^{*} V_{a}=\sqrt{2^{*} T-1} \\
& { }^{*} V_{r}=\frac{{ }^{*} T}{\sqrt{2^{*} T-1}} \\
& { }^{*} U=\frac{1-^{*} T}{\sqrt{2^{*} T-1}} \\
& { }^{*} \dot{m}=\frac{{ }^{*} T}{\sqrt{2^{*} T-1}} \\
& { }^{*} \omega=\frac{\left(1-{ }^{*} T\right)}{\sqrt{2^{*} T-1}} \\
& { }^{*} \dot{W}=\frac{2^{*} T\left(1-{ }^{*} T\right)}{\sqrt{2^{*} T-1}} \\
& \eta=2\left(1-{ }^{*} T\right)
\end{aligned}
$$

Equations (24) to (30) can provide the complete characteristics of the idealized simple frictionless reaction water turbine working at any condition. 
The universal characteristics of this turbine are presented graphically in Fig. 6 using the dimensionless torque ${ }^{*} T$ as the independent variable. Reducing the load torque applied to the machine from the stationary to runaway condition corresponds to moving from right to left on the horizontal axis.

Fig. 7 shows ${ }^{*} V_{a},{ }^{*} \dot{m},{ }^{*} T,{ }^{*} W, \eta$ presented as a function of ${ }^{*} \omega$. Although presented as a function of ${ }^{*} \omega$ the values shown were generated from equations (24) to (30) by varying ${ }^{*} T$. Equations (24)- (30), along with the graphical presentation in Fig.6 and Fig. 7 , offers the complete set of information in a universal form for the analysis of simple ideal outward flowing reaction water turbines.

\section{Discussion of ideal performance curves}

At the right hand end of the graphs in Fig. 6 the turbine is stationary and, as has already been discussed the mass flow rate through the turbine has its least value when the machine is not rotating. At this point the stationary torque $T_{s}$ given by Eq. 16 needs to be applied to balance the torque from the reaction to the flow of water $\dot{m}_{s}$ through the nozzles given by Eq. 9. At this point the value of non-dimensional torque ${ }^{*} T$ defined in Eq. 17 is equal to one (i.e. ${ }^{*} T=1$ ). Since the machine is stationary i.e. ${ }^{*} \omega=0$, no power is produced $\left({ }^{*} \dot{W}=0\right)$ and efficiency $\eta=0$. Furthermore $V_{a}$ is at its maximum $\left({ }^{*} V_{a}=l\right)$. 


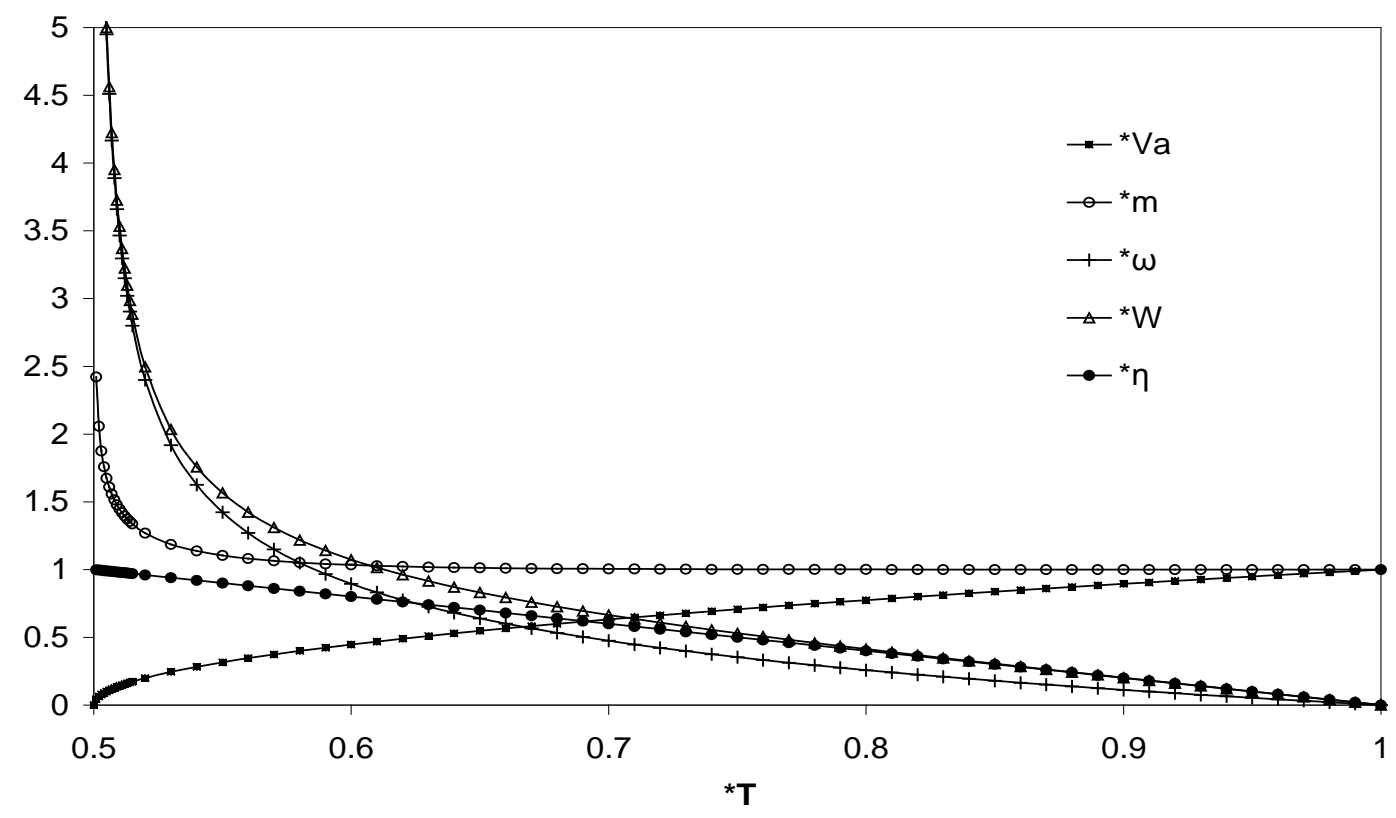

Figure 6 Graphical representations of parameters ${ }^{*} V_{a},{ }^{*} \dot{m},{ }^{*} T,{ }^{*} \dot{W}, \eta$ as a function of non dimensional torque ${ }^{*} T$

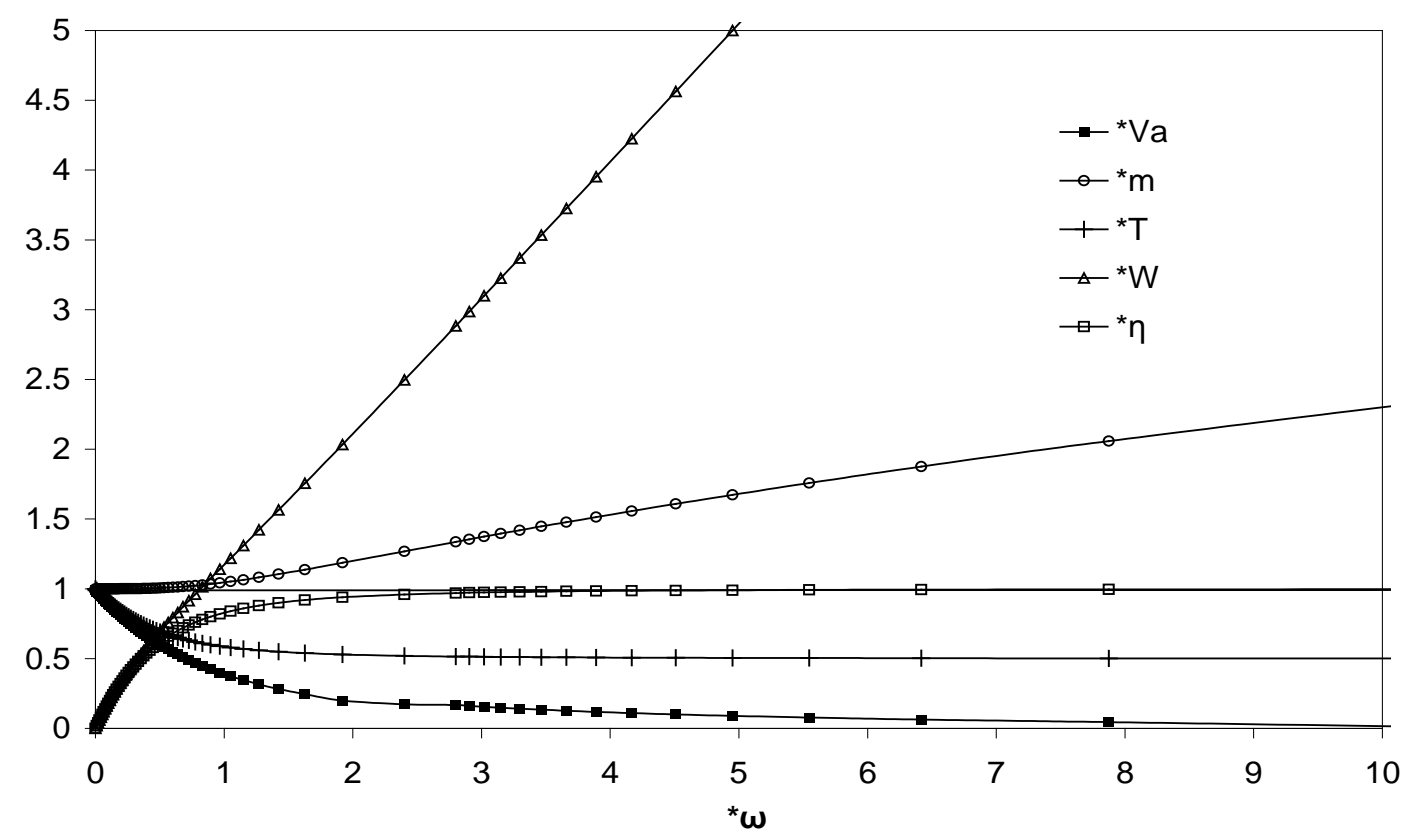


Figure 7 Graphical representations of parameters ${ }^{*} V_{a},{ }^{*} \dot{m},{ }^{*} T,{ }^{*} \dot{W}, \eta$ as a function of non dimensional angular velocity ${ }^{*} \omega$

If the turbine is allowed to start rotating by reducing the load torque, mass flow rate increases due to the centrifugal effect. Start of rotation is also accompanied with production of power ${ }^{*} \dot{W}$, increase of efficiency $\eta$ and a decrease in absolute velocity $V_{a}$, (hence ${ }^{*} V_{a},<1$ ).

Apart from efficiency $\eta$ which is a linear function of ${ }^{*} T$, the rates of change of the other functions,

${ }^{*} V_{a},{ }^{*} \dot{m},{ }^{*} \omega$, and ${ }^{*} \dot{W}$ increase with decrease of ${ }^{*} T$. The rate of change (as evident in equations (24) - (29) and shown in Fig 6) substantially increases and approach infinity as ${ }^{*} T$ approaches the value of 0.5 . The most efficient case will of course be when no absolute kinetic energy is left in the exiting stream, i.e. $V_{a}=0$. This occurs when ${ }^{*} T=0.5$, i.e. when the load torque to hold the turbine stationary is 0.5 . At this point mass flow rate of water through the turbine is infinite and the machine is producing infinite power. The speed of rotation at this point is also infinite. In reality of course windage and other frictional effects on the spinning turbine and friction on the fluid within the turbine prevent either $\omega$ or $\dot{m}$ reaching infinity but the trends shown by the analysis of the ideal machine is illuminating.

As seen from the analysis, the runaway speed of the turbine under consideration happens at ${ }^{*} T=0.5$ and not at $T=0$. This is contrary to the statements and conclusions made by Daugherty and Ingersoll [11] that associate zero torque with the infinite speed condition. 
Behavior of the turbine as ${ }^{*} T$ approaches 0.5: Under this condition the behavior of the three variables ${ }^{*} \dot{m},{ }^{*} \omega$ and ${ }^{*} \dot{W}$ is interesting. Considering the equations (27), (28) and (29) these three functions approach infinity with closely similar values. As a numerical example at ${ }^{*} T=0.501$ we have ${ }^{*} m=11.203,{ }^{*} \omega=11.158$ and ${ }^{*} \dot{W}=11.180$ as calculated from the corresponding equations. At this point ${ }^{*} V_{a}=0.045$ and $\eta=0.998$.

At ${ }^{*} T=0.5001$ the values of ${ }^{*} \dot{m},{ }^{*} \omega,{ }^{*} \dot{W}$ are $35.362,35.348$ and 35.355 respectively. At this point ${ }^{*} V_{a}=0.0014$ and $\eta=1.000$.

\section{Discussion of the general characteristics}

Based on the analysis presented in the previous discussions it is seen that the idealized simple reaction water turbine is more efficient the faster it turns. Moreover, the centrifugal effect which pushes more water through the turbine results in an increase in the power producing capacity of the machine. This can possibly be important as it may enable production of compact water turbine of small sizes. Also the fact that the above machine achieves its highest efficiency at high speeds can also result in smaller and more compact electrical generators. Since the machine increases its volume throughput by using the centrifugal pumping effect, this may mean that the simple ideal reaction water turbine could offer a solution for power production from water reservoirs of low head which may not be suitable to be utilized by conventional water turbines operating on the impulse principal. The runaway potential at low loads however must be recognized. More sophisticated inward flowing reaction turbine types such as the Thomson and Francis have some self-governing effect in that high speeds of rotation cause an increase of centrifugal pressure that opposes the inward flow. 


\section{Example}

Here through some numerical examples some of the points made above are demonstrated. Assume the rotor diameter to be $0.25 \mathrm{~m}$ having two nozzles each having an exit opening of $10 \mathrm{~mm} \times 10 \mathrm{~mm}$. This results in total nozzle exit area $A$ of $2 \times 10^{-4} \mathrm{~m}^{2}$. The density of water is assumed to be $\rho=1000 \mathrm{~kg} / \mathrm{m}^{3}$ and acceleration of gravity $g=9.81 \mathrm{~m} / \mathrm{s}^{2}$. The characteristics of such a rotor i.e. torque, power and efficiency are presented in Fig. 8 to Fig. 10 as functions of rotational speed for four different heads namely $1 \mathrm{~m}, 2 \mathrm{~m}, 5 \mathrm{~m}$ and $10 \mathrm{~m}$. This is the range of water heads which are considered to be more suitable for simple reaction water turbines. It is in this range that it is believed at this stage that the above turbine has its most applications.

As shown previously, Fig. 5 presents the variation of water flow rate versus rotational speed. It is observed that for the same head, flow rate increases with rotational speed. The rate of increase is initially low, but it increases at higher rotational speeds and eventually assumes a linear variation. It is interesting to note that the effect of the head on water flow rate diminishes at higher speeds. This is due to the self-pumping phenomenon, which is as explained before, one of the main features of the machine under discussion. From the graphs in Fig. 5 it is evident that the curves relating to different heads become closer and follow an asymptote as rotational speeds increases.

Fig. 8 shows the variation of shaft torque versus rotational speed. As seen for all four heads the maximum torque is produced when the machine is not rotating. The rotation of the machine is accompanied with a drop in the produced torque. The torque is decreased further as speed increases approaching asymptotically half the value of the starting torque. 


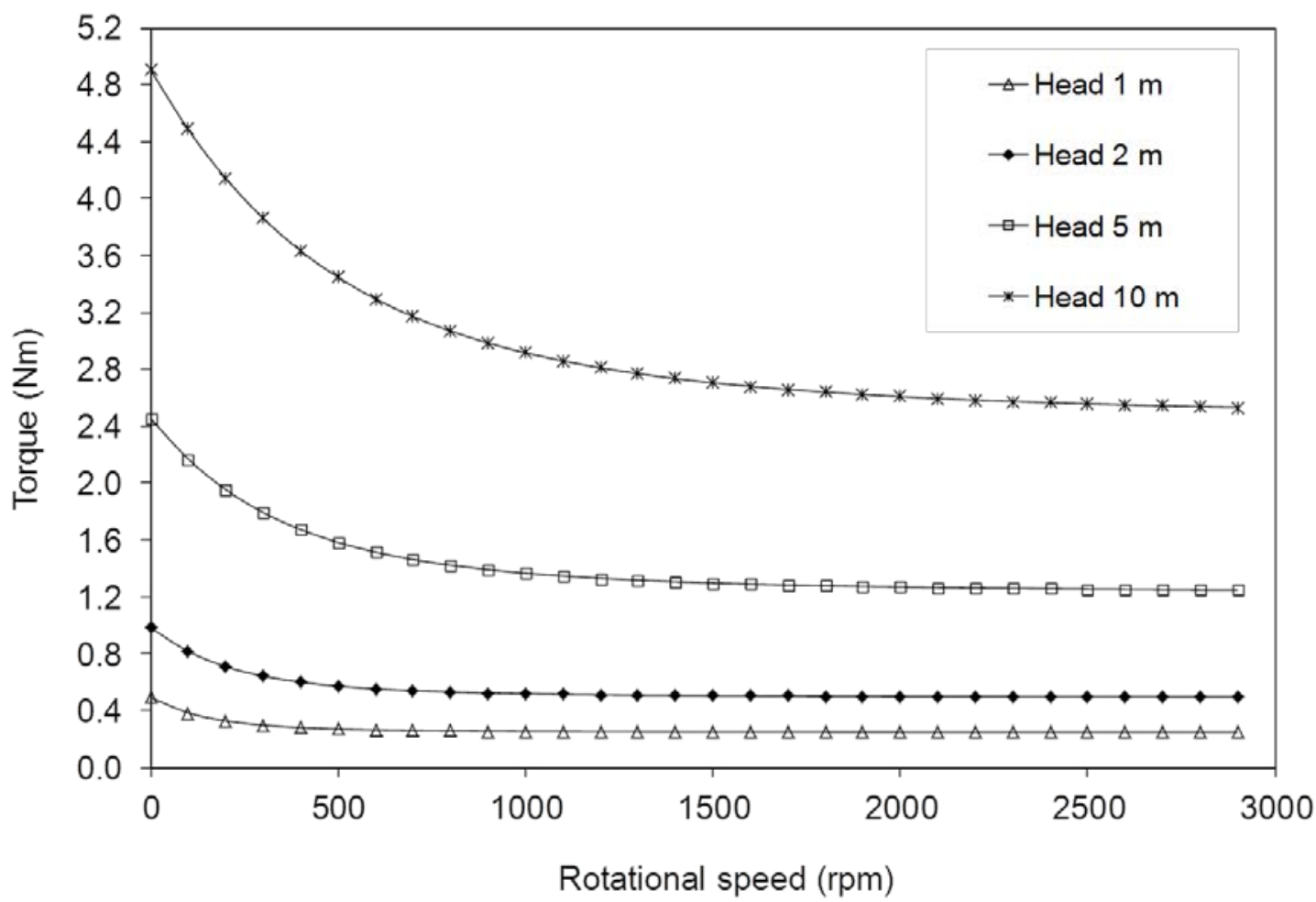

Figure 8 Variation of shaft torque with speed for several supply heads

Fig. 9 shows the power versus rotational speed. The speed here is limited to $3000 \mathrm{rpm}$ for practical considerations. It is seen that for the same head the power increases with rotational speed. This variation is almost linear over most of the range.

Fig. 10 shows the variation of efficiency versus rotational speed. It is seen that for the same water head initially the efficiency increases substantially with the speed and approaches 1 asymptotically as speed approaches infinity. It is also shown that for the same rotational speed the machine operates at higher efficiency when applied to lower heads. This makes the machine especially attractive for application to production of power from low head reservoirs, for example in certain types of tidal power plant. 


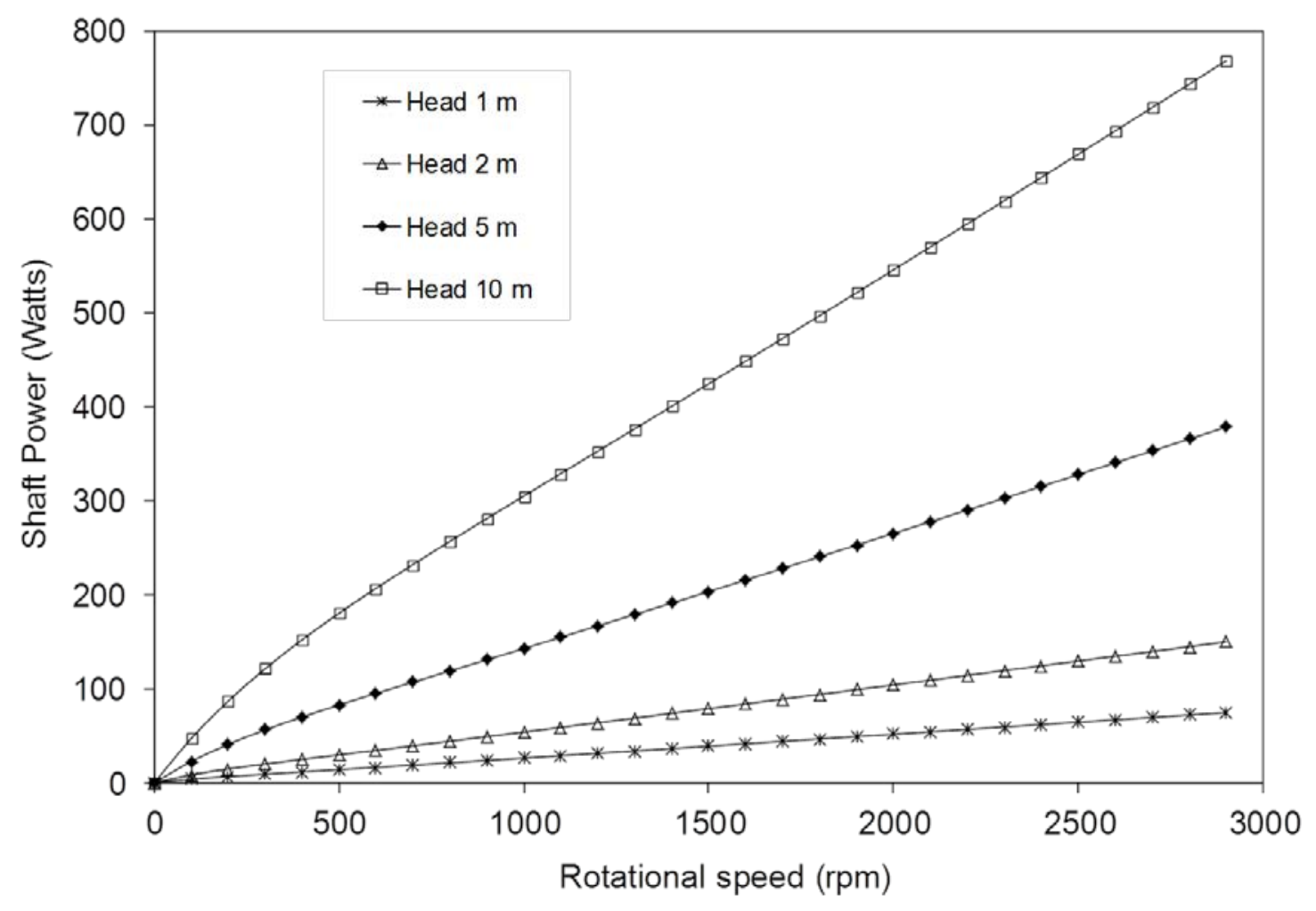

Figure 9 Variation of power with speed for several supply heads

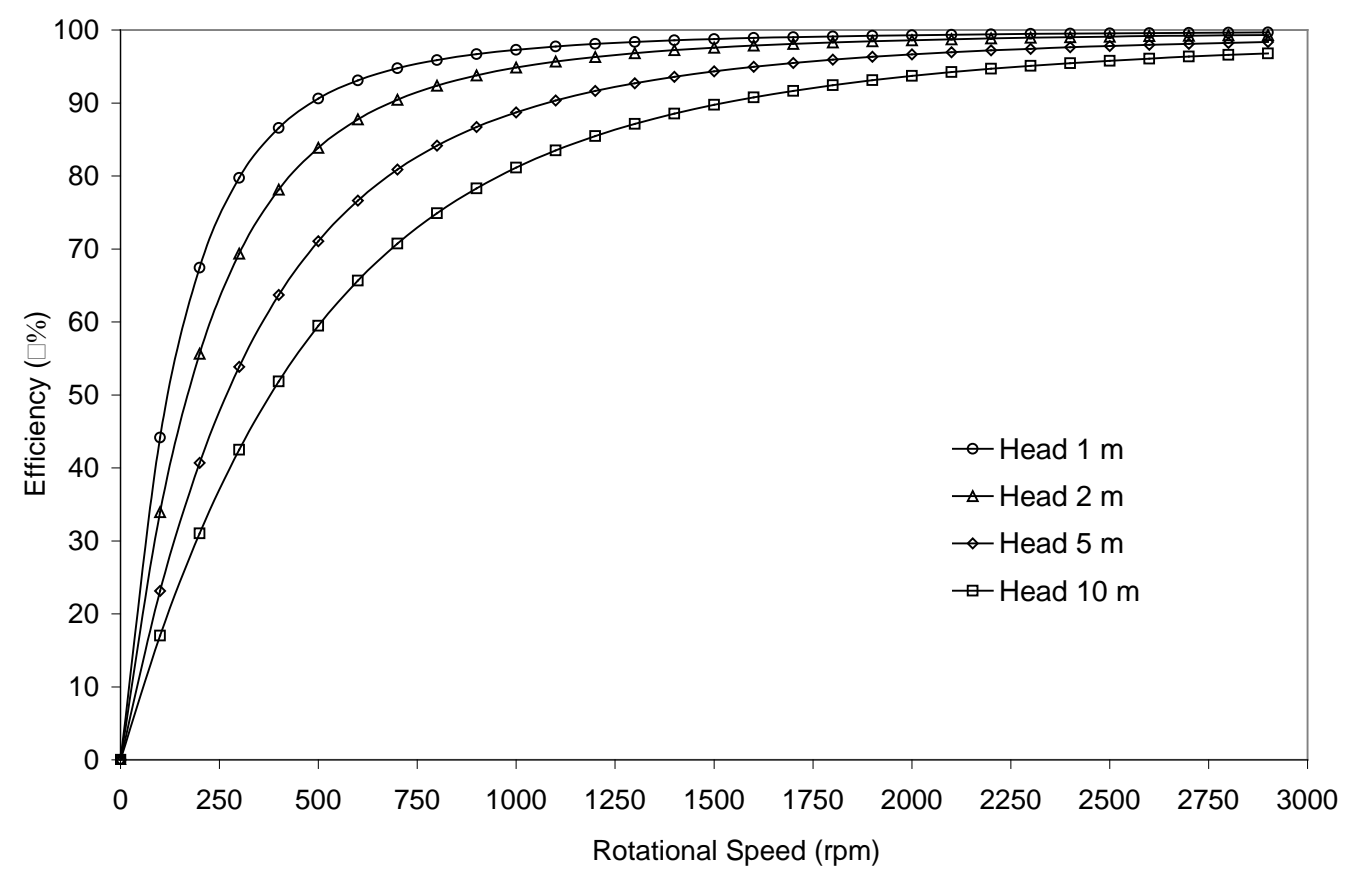


Figure 10 Variation of efficiency with the speed for several supply heads

Information provided in Figs. 5, 8, 9 and 10 is presented in different form in Figs. 11 to 13. In these figures it is assumed that the rotor is coupled to an induction motor having 2 , 4 or 8 poles and operating as electrical generator. In this case when the machine rotates at $3000 \mathrm{rpm}, 1500 \mathrm{rpm}$ and $750 \mathrm{rpm}$ then the frequency of the alternating current will be 50 Hz. In Fig. 11 through 13 the mass flow rate, torque and power are presented as functions of water head for 3 different rotational speeds namely 750 rpm, $1500 \mathrm{rpm}$ and $3000 \mathrm{rpm}$ These figures would enable estimation of the electrical output of the described ideal rotor when applied to different reservoir heads of up to $10 \mathrm{~m}$ water.

Fig. 11 shows the variation of water flow rate as function of head. As seen from this figure for the same head the water flow rate is higher when the machine rotates faster. It is also interesting to note that the water flow rate is generally a weak function of head and for the case of rotational speed of $3000 \mathrm{rpm}$, the water flow rate is almost independent of the water head. This point again to the fact that the self pumping effect at higher rotational speeds dictates the flow rate and the effect of the head become minimal. When the driving head is zero flow due to the centrifugal pumping effect could be made to occur by rotating the rotor with some driving device. 


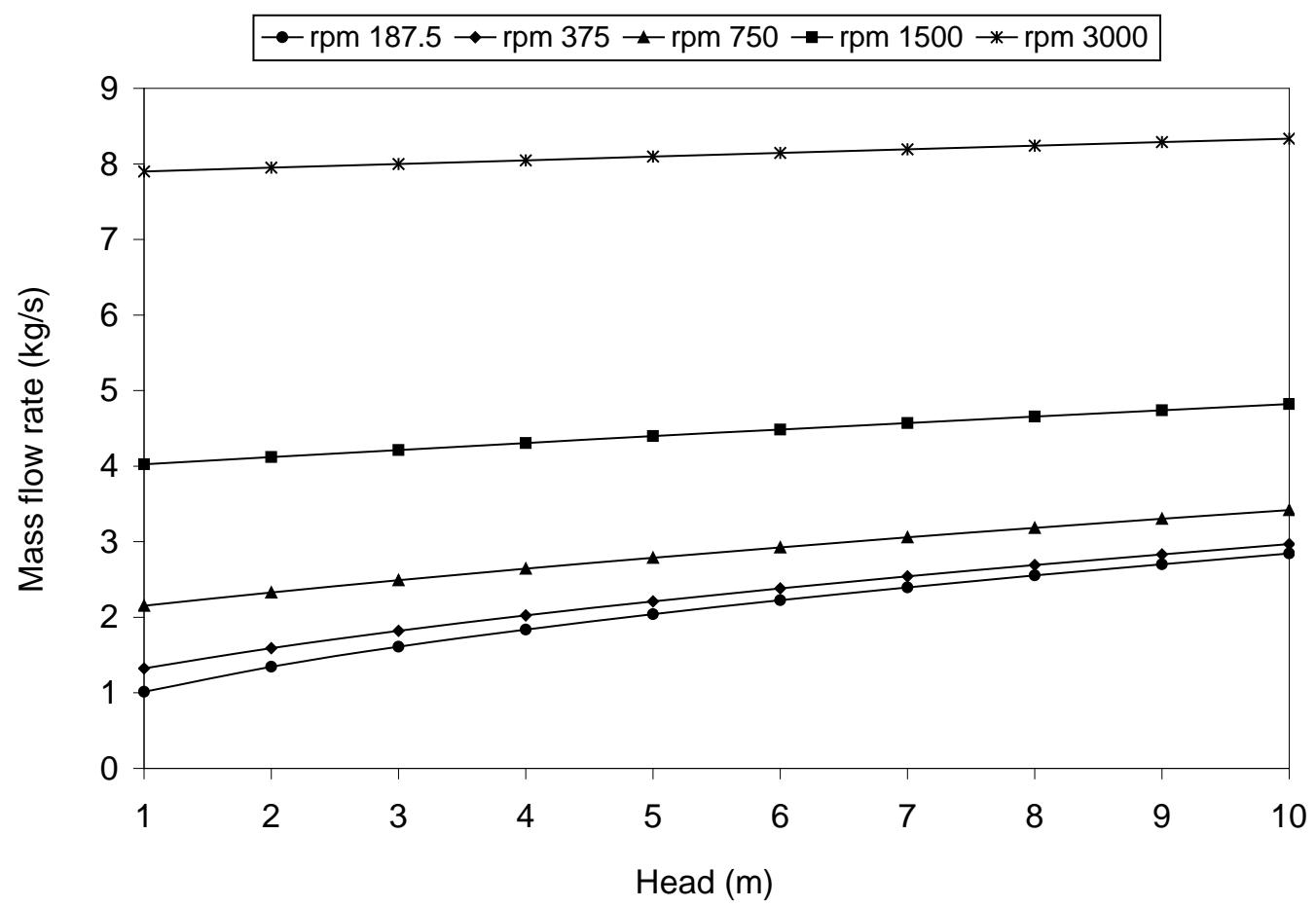

Figure 11 Variation of mass flow rate with rotation speed for various supply heads

Fig. 12 shows the shaft torque produced by the rotor as a function of the head of water. It is seen that for the same head a faster machine has lower torque. However it can be observed that the rotational speed does not have a major effect on the torque. In other words at $750 \mathrm{rpm}$, or $1500 \mathrm{rpm}$ or $3000 \mathrm{rpm}$ the machine have more or less the same torque when applied to the same water heads in the lower part of the range under investigation.

Fig. 13 is a clear indication that the output power from the same machine increases almost linearly when applied to higher heads. Therefore this gives a simple rule for predicting the capacity of the machine operating under different heads. Assuming the applied load is such that the rotational speed is maintained constant. In Fig. 13 it is also seen that the capacity of the machine is higher for higher values of rotational speed. 


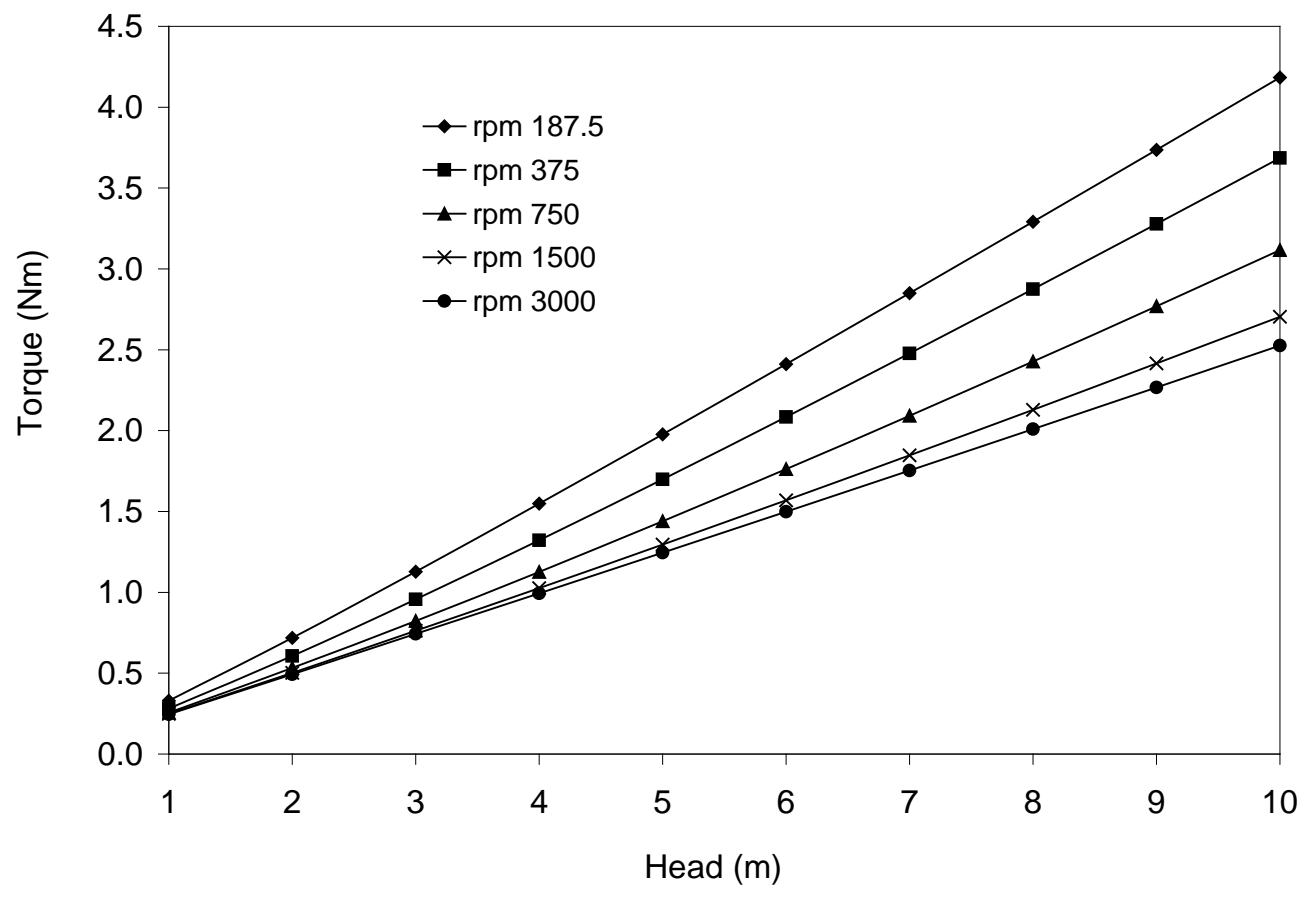

Figure 12 Variation of torque with rotational speed for various supply heads

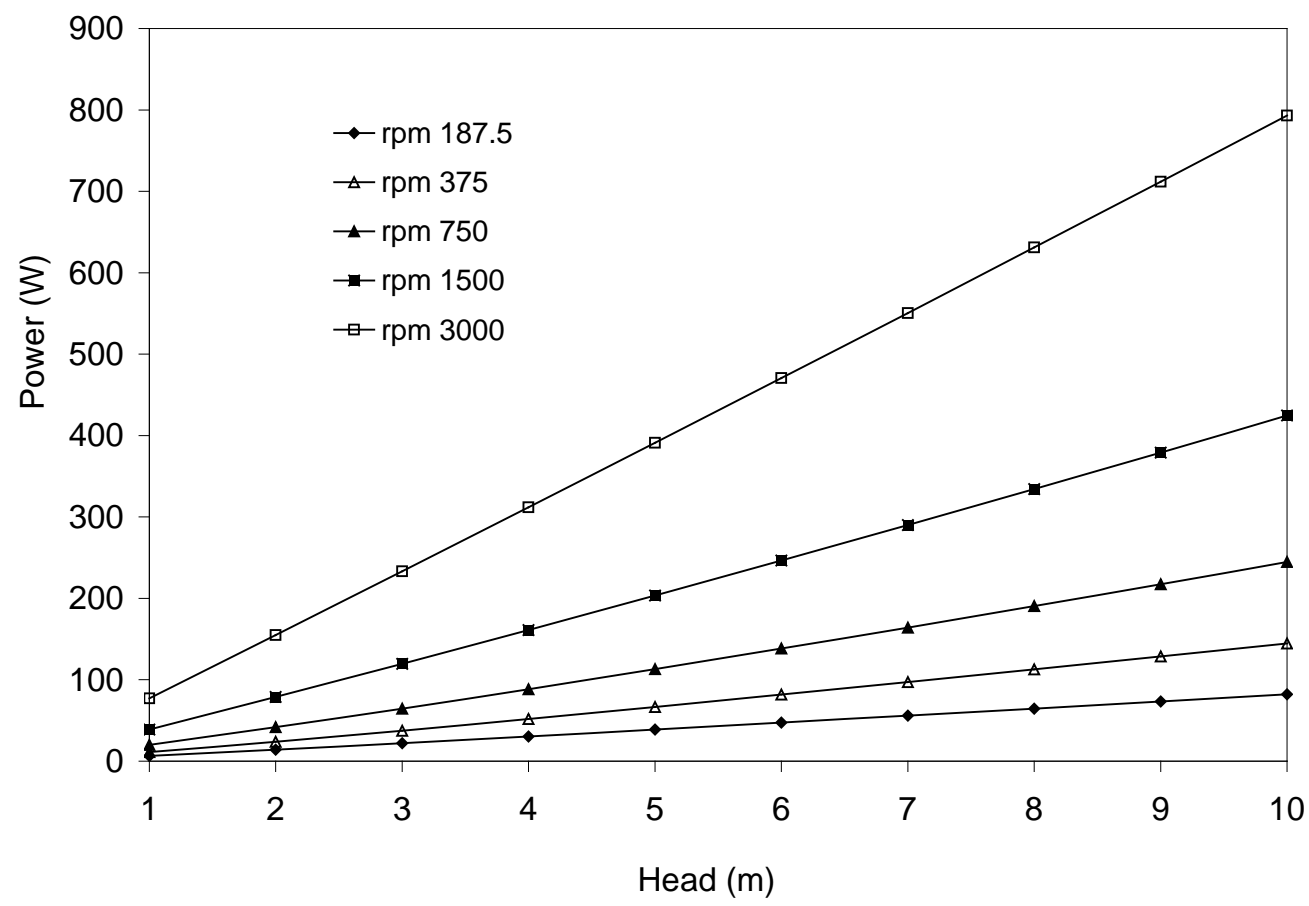

Figure 13 Variation of power with supply head for various rotational speeds 
Simple reaction turbine and specific speed

Specific speeds are commonly used as a tool for comparison of the characteristics of similar hydraulic machines. Here this tool is applied to simple reaction water turbine, by using the formulation provided by Turton [12] for the specific speed of turbines, the following relation between specific speed and efficiency of a simple reaction turbine has been derived.

$$
K_{s}=2^{-3 / 4} \times \frac{\sqrt{A}}{R} \times \frac{\eta \sqrt{2-\eta}}{(1-\eta)^{3 / 4}}
$$

The effect of geometry on the relation between specific speed and efficiency is expressed in terms of $\sqrt{A} / R$. However this can be changed to ratio of diameters i.e. $D / d$ where $D=2 R$ and $d$ is the diameter of the nozzle. Defining an equivalent exit nozzle diameter $d_{e}$ as,

$$
d_{e}=\frac{2}{\sqrt{\Pi}} \times \sqrt{A}
$$

Using the relation for the equivalent exit nozzle diameter from equation (2), we can rewrite equation (1) as follows,

$$
K_{s}=2^{-3 / 4} \times \sqrt{\Pi} \times \frac{d_{e}}{D} \times \frac{\eta \sqrt{2-\eta}}{(1-\eta)^{3 / 4}}
$$




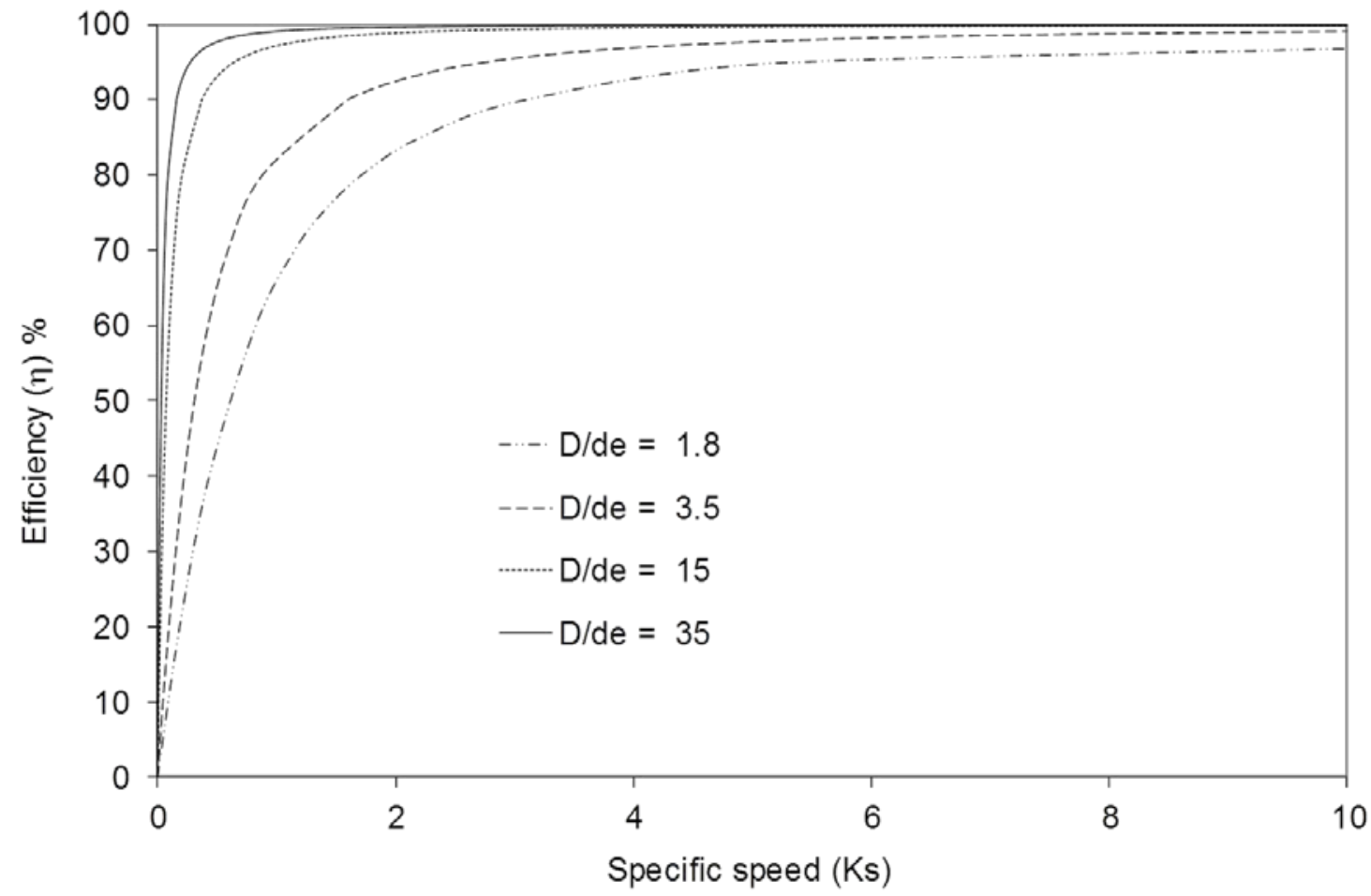

Figure 14 Variation of efficiency with specific speed for various diameter ratios

It is not possible to express $\eta$ as a function of $K_{s}$ in an explicit form. However the variation of $\eta$ as a function of $K_{s}$ is presented in Fig. 14 for several values of diameter ratios. It is seen in Fig. 14 that simple reaction machines achieve higher efficiencies for machines of higher diameter ratios $\left(D / d_{e}\right)$ i.e. for a given diameter $\mathrm{D}$ a smaller nozzle exit area will improve efficiency. Since we can relate the capacity of the machine to the nozzle area we can then say that for the same specific speeds and rotor diameters, machines of smaller capacities would operate at higher efficiency (Inclusion of consideration of frictional effects may of course cause such conclusions to be revised). From the same figure it can be also concluded that efficiency of simple reaction machines improves as the specific speed $K_{s}$ increases. Considering the definition of $K_{s}$ in Eq. 33 
this is equivalent to saying high efficiencies are achieved at high speeds, high volume flow rate and low heads. This can be considered an important conclusion in relation to the characteristics of simple reaction water turbines.

\section{Analysis of simple reaction water turbine for practical operating condition with losses}

Now if we consider real operating condition there would be considerable amount of losses associated with the flow of water through the simple reaction water turbine. In this section a factor that would represent the losses associated with the fluid flow through the turbine has been defined, this factor would be called as $k$-factor throughout this paper.

For a practical situation the energy balance equation would be different than that in an ideal situation, as there would be some energy lost due to fluid friction within the turbine and a majority at the exit nozzles due to the high velocity of exiting water jets. So in this situation the energy balance equation would be written as follows,

$$
\dot{m} g H=\dot{W}+\frac{1}{2} \dot{m} V_{a}^{2}+\frac{1}{2} \dot{m} k V_{r}^{2}
$$

Here, $\frac{1}{2} \dot{m} k V_{r}^{2}$ represents the fluid frictional losses associated with the flow of water through the turbine exit nozzles, $\frac{1}{2} \dot{m} V_{a}^{2}$ represents the loss of kinetic energy with the water leaving the turbine, $\dot{W}$ represents the shaft power and $\dot{m} g H$ is the rate of potential energy supplied to the turbine. So with increase in the value of $k$-factor the shaft power would decrease and this is obvious from the following equation, 


$$
\dot{W}=\dot{m} g H-\frac{1}{2} \dot{m} V_{a}^{2}-\frac{1}{2} \dot{m} k V_{r}
$$

Following equation for the relative velocity is derived by re-arranging the above equation,

$$
V_{r}=\sqrt{\frac{1}{(1+k)}} \times \sqrt{2 g H+R^{2} \omega^{2}}
$$

In a situation where $k$-factor of the exit nozzle is known the relative velocity can be estimated without knowing the exit nozzle area. Further an equation for k-factor has been derived below from the relative velocity equation. This equation can be used to estimate the actual k-factor of a turbine using the experimental data.

$$
k=\frac{2 g H+R^{2} \omega^{2}}{\left(V_{r}\right)^{2}}-1
$$

Here $V_{r}=\frac{\dot{m}}{\rho A}$ is estimated from the experimentally measured value of mass flow rate, measured exit nozzle area and density of water. From the above equations we can say that if we keep the supply head constant then the $k$-factor will be a function of angular speed and the relative velocity. Consider an example, where water is flowing through a stationary nozzle with constant exit area, here the fluid friction losses ( $k$-factor) for this nozzle would be proportional to the relative velocity of the exiting fluid and relative velocity would depend upon supply head.

Similarly in case of rotating nozzle the fluid friction losses ( $k$-factor) would be proportional to the relative velocity or mass flow rate of the exiting fluid. While here if 
the turbine is rotating then the relative velocity / mass flow rate would change with change in angular speed due to centrifugal pumping effect discussed earlier.

\section{Torque, mass flow and rotational speed characteristics}

Fig. 15 shows the variation of torque $T$ and mass flow rate $\dot{m}$ with respect to the rotational speed for a turbine with rotor radius $R=0.125 \mathrm{~m}$, the total exit area $A=2 \times 10^{-4}$ and an assumed k-factor of 0.05 . After considering the fluid frictional losses associated with the flow of water through the turbine exit nozzles it can be seen from Fig. 16 that the turbine will not experience runaway condition in real operation due to the power loss in the turbine. Further it can also be seen that as the load torque is decreased the angular speed of the turbine will increase causing the mass flow rate of water flowing through the turbine to increases but will never reach a value close to infinity.

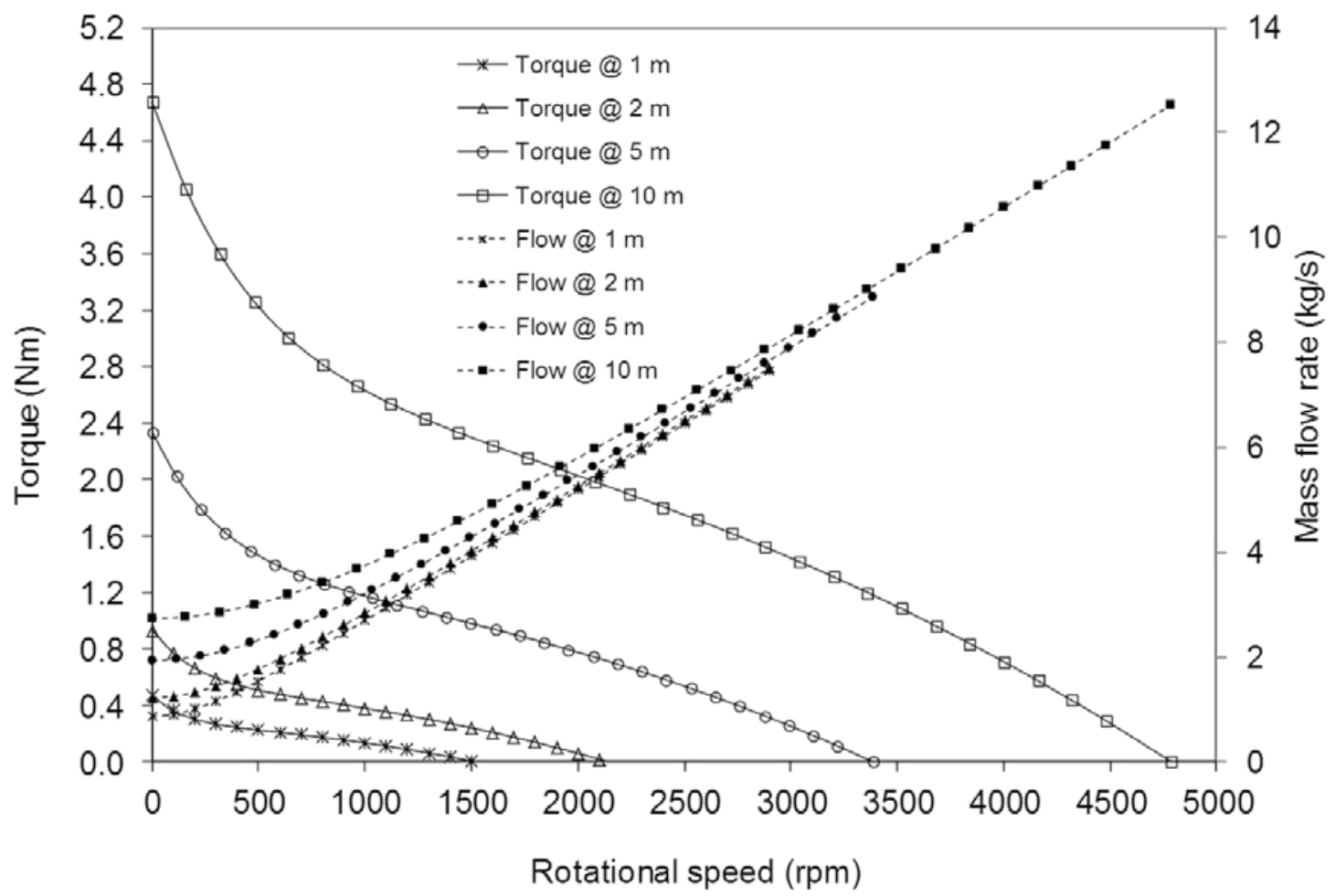

Figure 15 Variation of torque with rotational speed at different supply heads 


\section{Power characteristics}

The power curves shown in Fig. 16 are predicted for a simple reaction water turbine with rotor radius of $R=0.125 \mathrm{~m}$, total nozzle exit area of $A=2 \times 10^{-4} \mathrm{~m}^{2}$ and an assumed kfactor of 0.05 . In reality in presence of losses the power produced by the turbine never reaches infinity as shown in Fig.16. The power curves for a simple reaction turbine model can be experimentally estimated by initially allowing the simple reaction turbine to rotate at its maximum possible speed under no external load and at constant supply head while all the parameters (flow rate, head, rotational speed and load torque) are recorded. Then gradually increase the load on the turbine in steps and record the parameters for each step, while the supply head is kept constant.

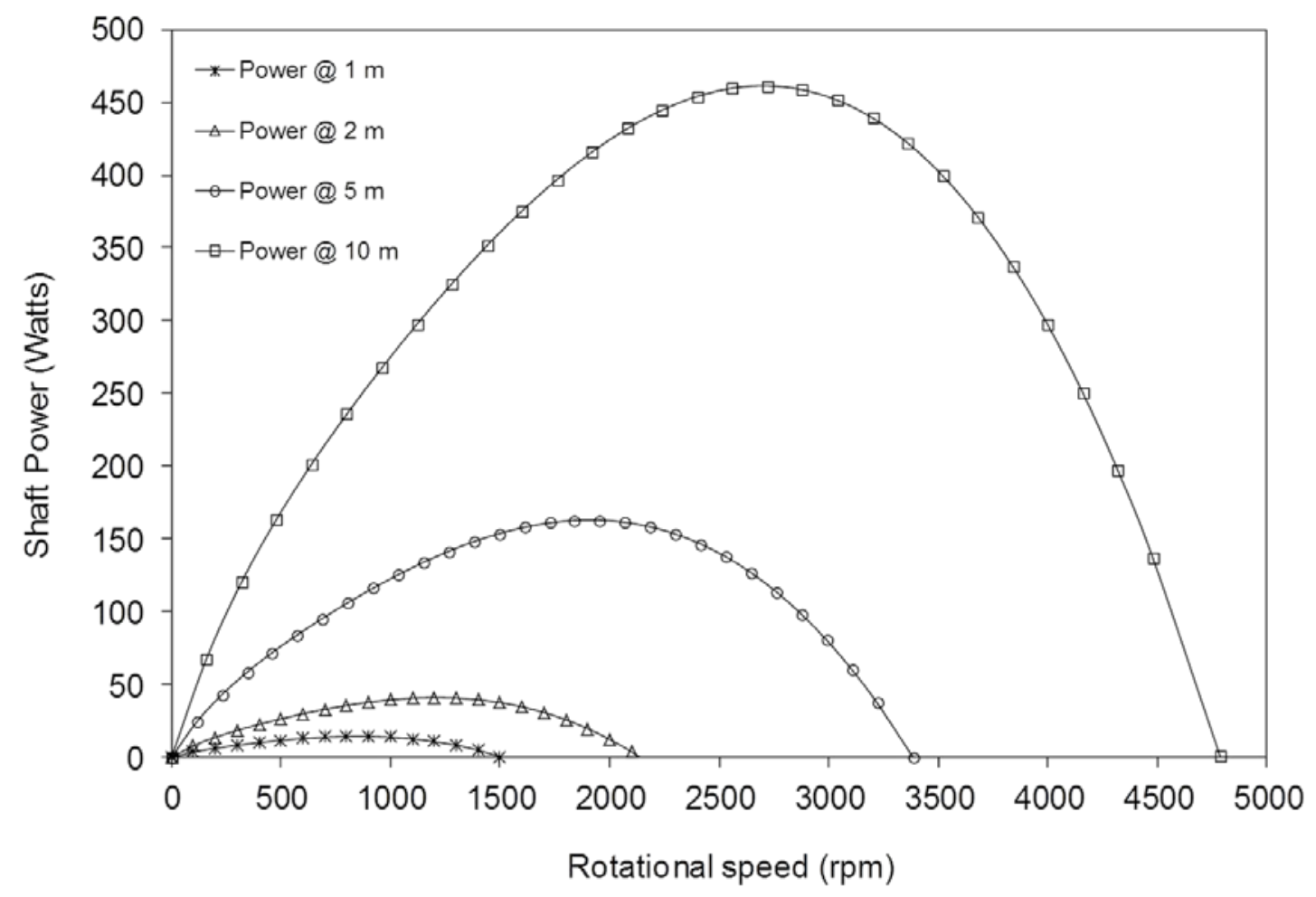

Figure 16 Variation of power with rotational speed at different supply heads 


\section{Effect of fluid friction on turbine efficiency characteristics}

From the analysis of ideal simple reaction turbine it was previously concluded that faster the turbine runs more efficient it will be and eventually reach a value of unity. But after the analysis of simple reaction turbine with fluid frictional losses it is seen that the maximum efficiency depends on the value of k-factor and would never reach unity as shown in Fig. 17. The predicted efficiency curves for a turbine with a rotor diameter of $0.12 \mathrm{~m}$ and assumed values of $\mathrm{k}$-factors operating at two different heads $1 \mathrm{~m}$ and $10 \mathrm{~m}$. For example, it can be seen that with a $k$-factor of 0.1 the maximum turbine efficiency at $1 \mathrm{~m}$ head and $10 \mathrm{~m}$ head is exactly the same independent of its corresponding rotational speed.

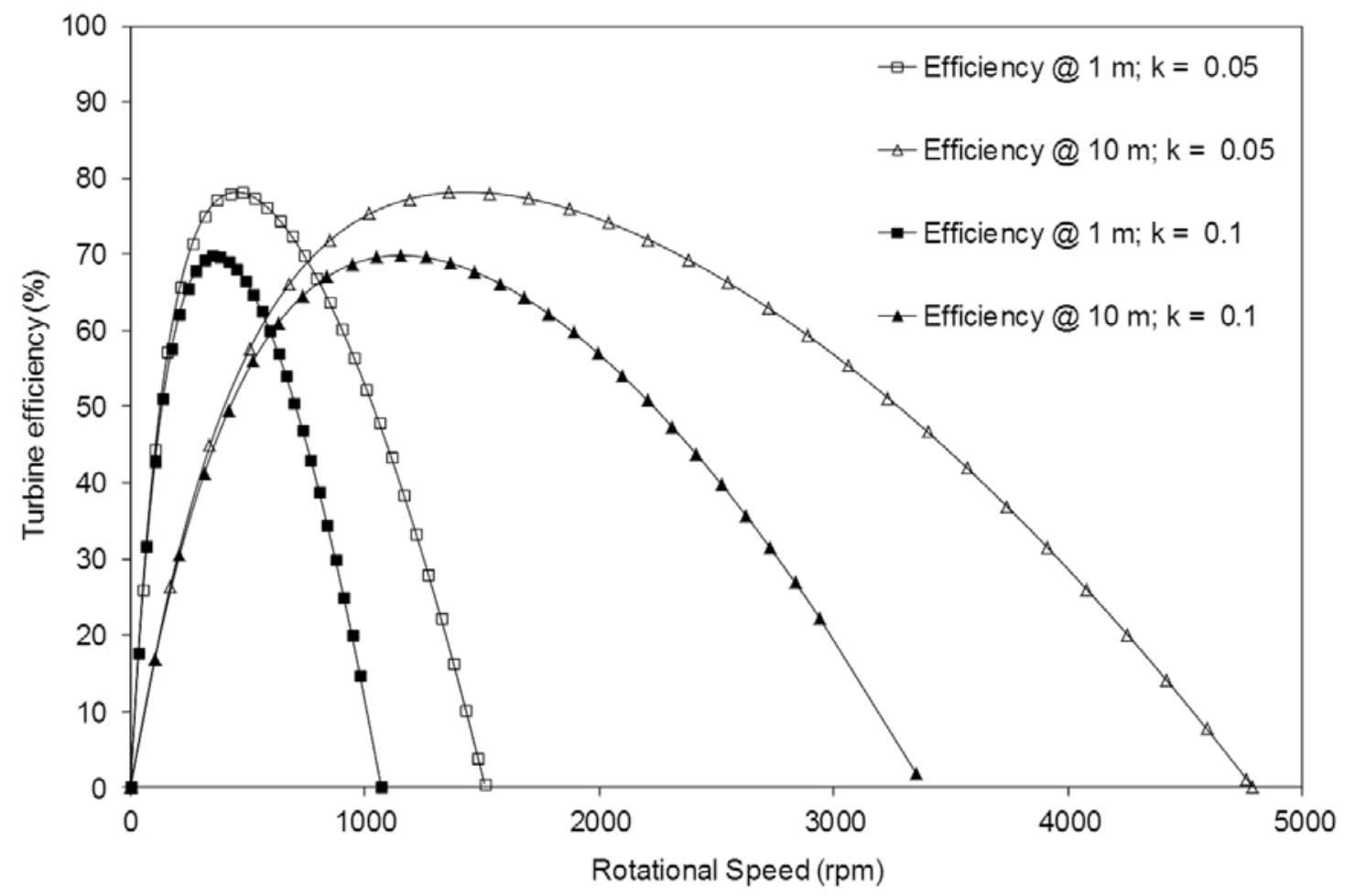

Figure 17 Effect of $k$-factor on turbine efficiency 


\section{Experimental analysis}

Experimental performance of two split reaction water turbine models shown in Fig. 18 has been discussed in this section $[13,14]$. First turbine model has a mean diameter of $240 \mathrm{~mm}$ and the second turbine model has a mean diameter of $120 \mathrm{~mm}$, while both these turbines have similar inlet and exit areas. Both the turbine models have two rectangular shape exit nozzles with $6 \mathrm{~mm}$ width and $120 \mathrm{~mm}$ length, i.e. total exit area of $1440 \mathrm{~mm}^{2}$. Fig. 19 and Fig. 20 shows the relationship between the measured/estimated power and the rotational speed, at the same time the flow rate is shown on the secondary y-axis. The measured values show actual measurements, e.g. measured electrical power is equal to the product of measured voltage and current output from the generator. Whereas the estimated values show the sum of measured power and power lost in friction. The estimated mechanical power loss represents the sum of power loss from the inlet lip seal, bearings and air drag. To estimate the sum of mechanical power loss the installed turbine is rotated using a DC motor at different rotational speeds. Here the power consumed by the DC motor at different rotational speeds represents the overall mechanical losses associated with the turbine and DC generator (motor) assembly. Overall it can be seen that for both the turbines the frictional power loss increases as the rotational speed increases. A closer look at the graphs shows that the mechanical power losses in turbine model 2 are less as compared to the mechanical power losses in turbine model 1 at a low rotational speed. For example, for turbine model 1 at 600rpm the mechanical power loss is about 58Watts, whereas for turbine model 2 at 600rpm the mechanical power loss is about 30Watts. This reduced mechanical power loss is a result of reduced air drag losses due to small rotor diameter of turbine model 2. From experiments it was observed that the 
air drag increases as the turbine exit nozzle width is increased, this was due to the shape of turbine drifting away from a perfect circle to more elliptical as the exit nozzle width is increased.

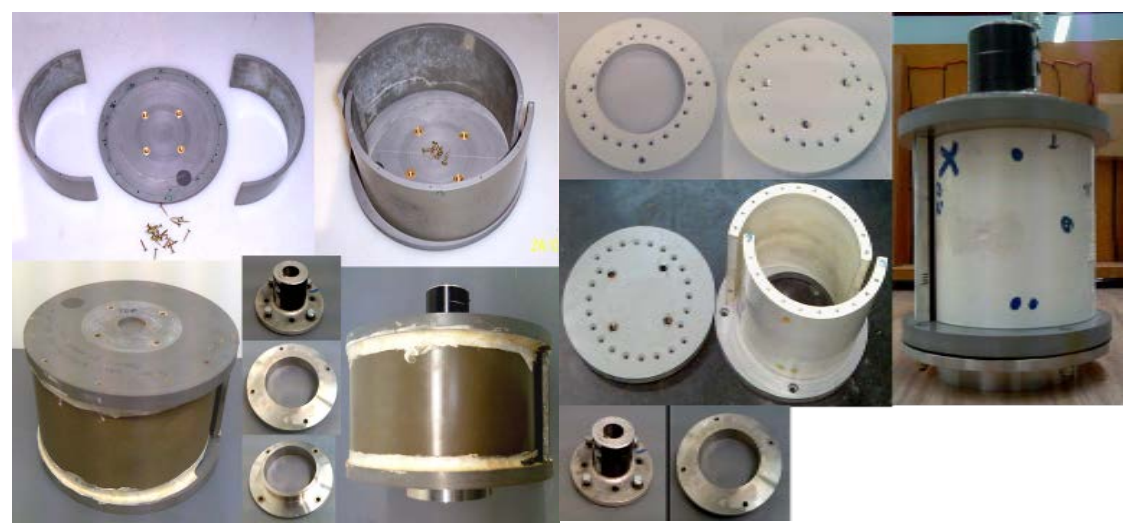

Turbine Model 1

Turbine Model 2

Figure 18 Picture of two split reaction water turbines

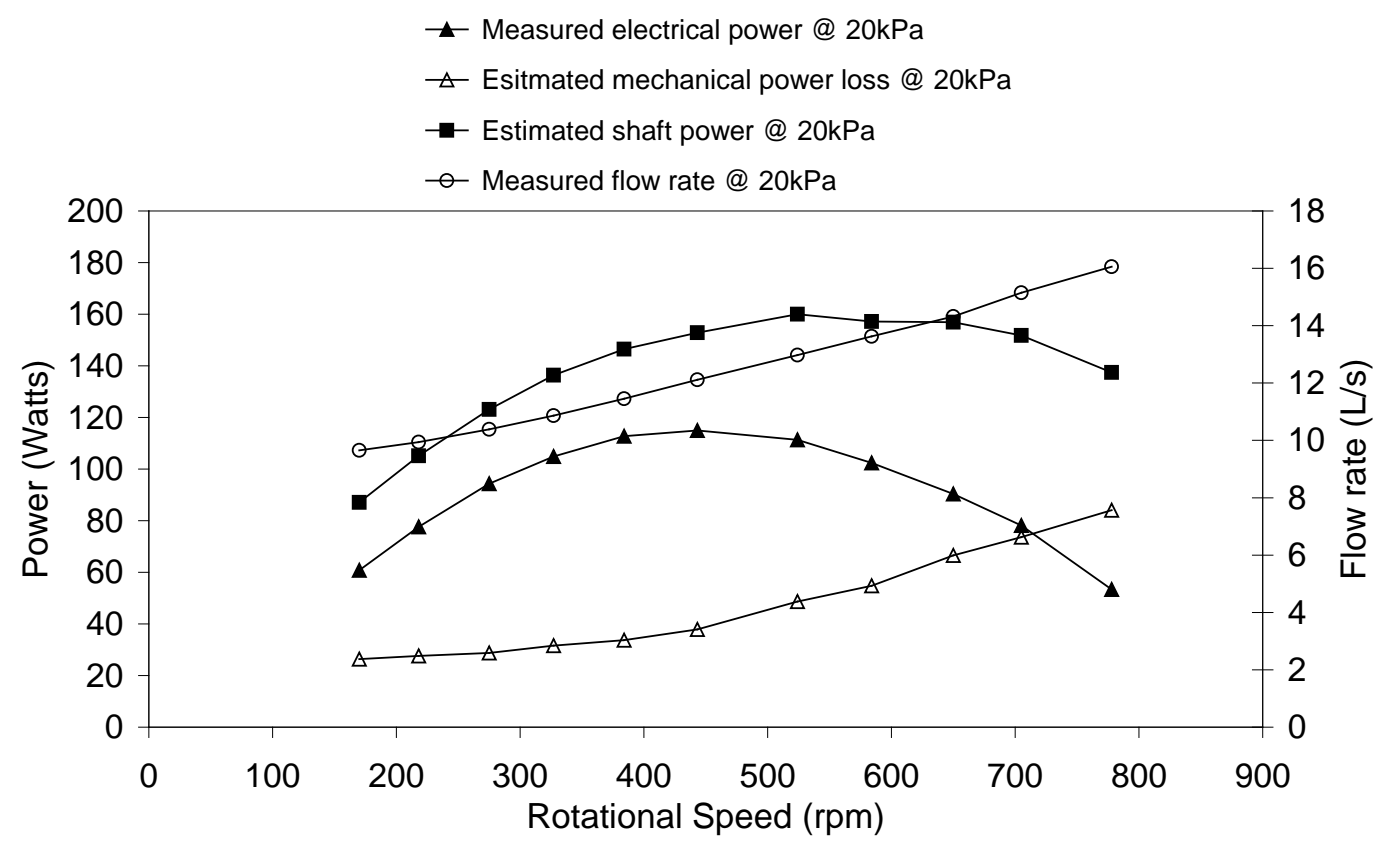

Figure 19 Measured and estimated performance for turbine model 1 (240mm diameter) 


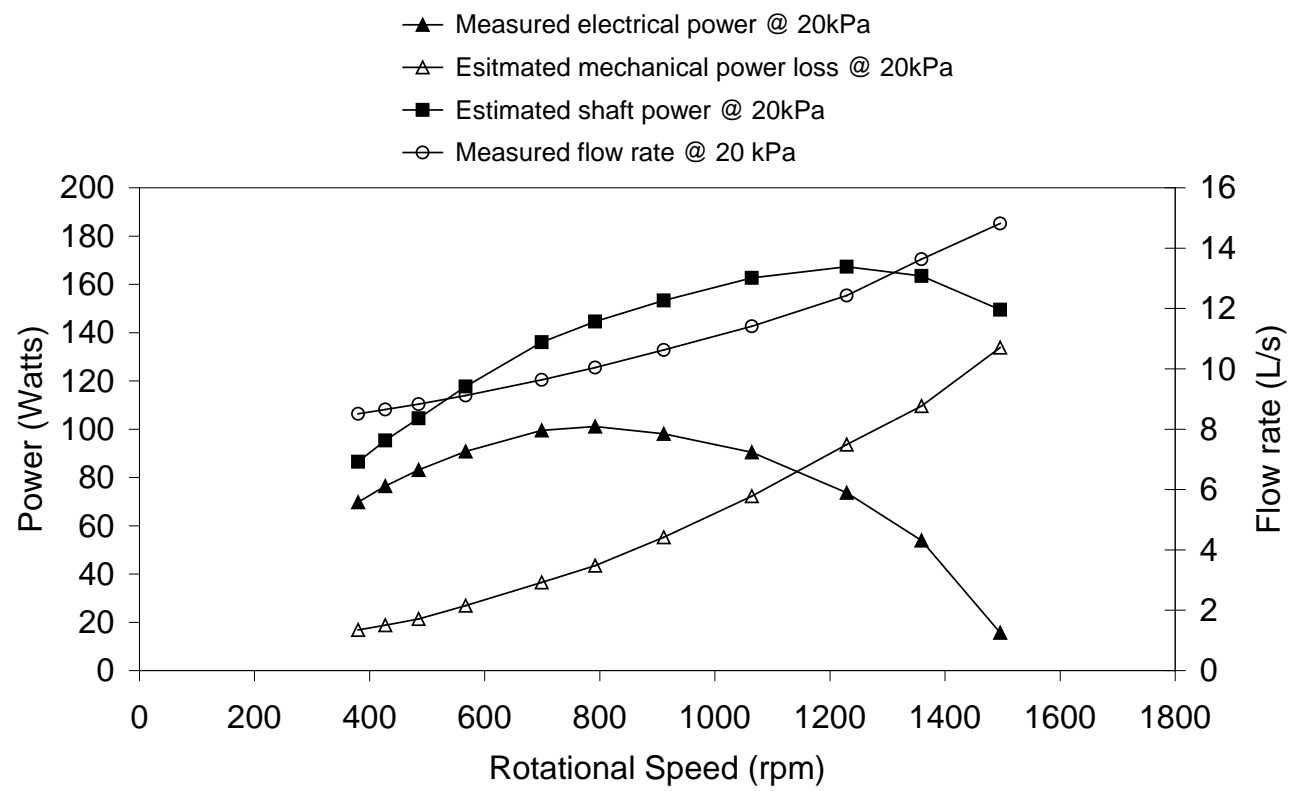

Figure 20 Measured and estimated performance for turbine model 2 (120mm diameter)

Fig. 21 shows the relationship between the turbine efficiency and the rotational speed. The turbine efficiency is defined as the energy conversion efficiency from potential energy in the water to mechanical energy at the turbine shaft. Shaft power which includes electrical power output plus the mechanical frictional losses as discussed earlier is used to estimate the turbine efficiency, so the inefficiency is only due to the fluid frictional losses within the turbine. It can be seen from the Fig. 21 that turbine model 2 has higher turbine efficiency then turbine model 1 and this can be contributed to the smaller diameter of the turbine model 2. The water that flows into the turbine is moving axially upwards, while the turbine inner surface is rotating. The different in the angular velocity of the solid surface and water inside the turbine adds internal water drag on the turbine. Turbine model 2 has less wet surface area due to the small diameter and hence has lesser internal water drag as compared to the turbine model 1. 


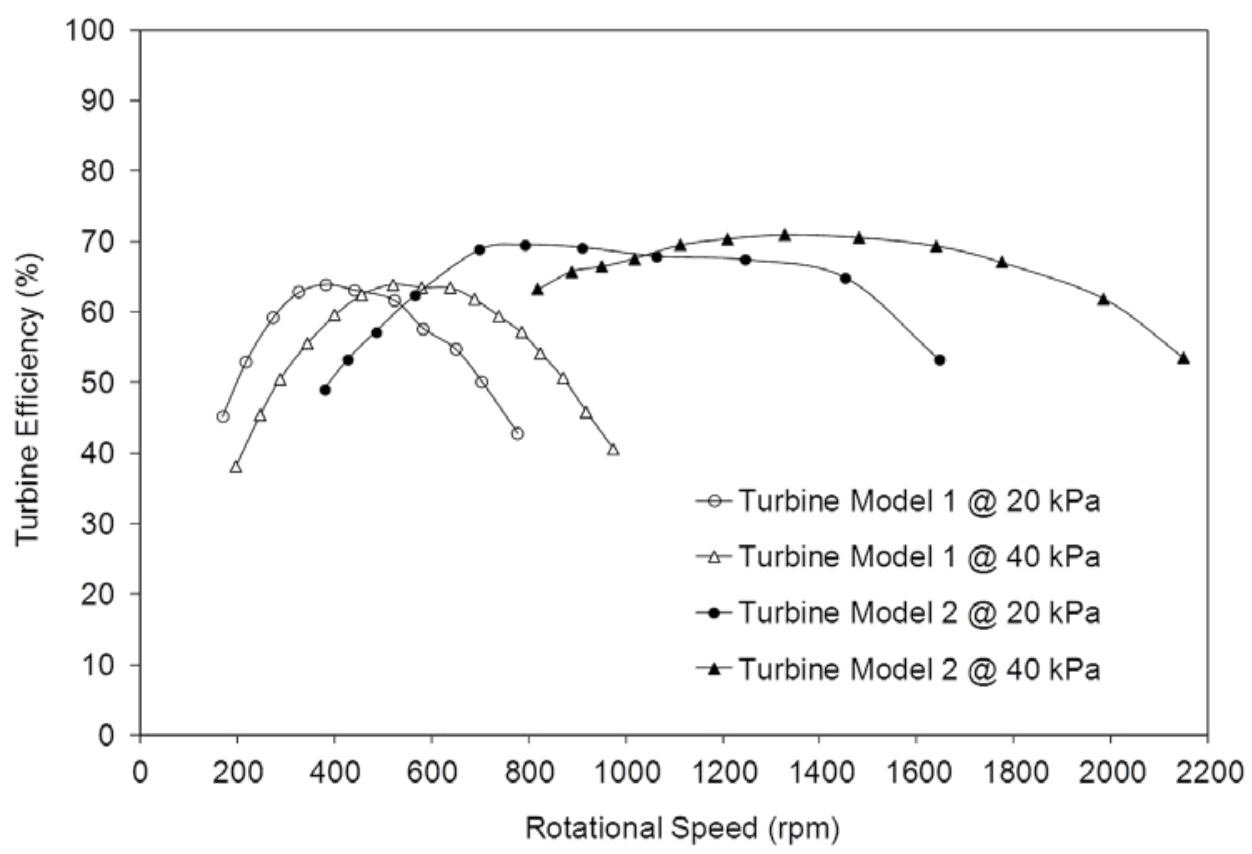

Figure 21 Estimated turbine efficiencies for model 1 and model 2

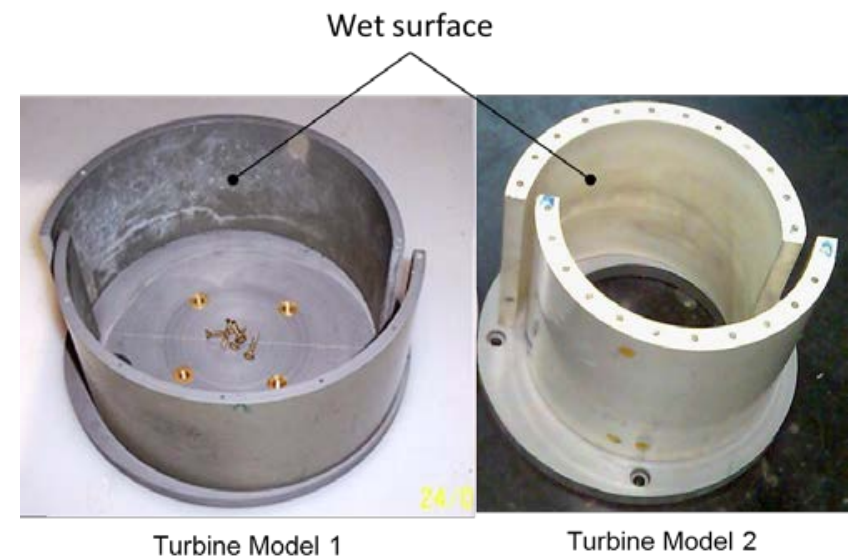

Figure 222 Wet surface area of turbine model $1 \& 2$

\section{Uncertainty analysis}

Uncertainty analysis of the measured and estimated parameters was carried out following the law of error propagation [15]. Following examples describe the procedure used to for estimating the uncertainty, 
The volume flow rate of the water flowing through the turbine was estimated by using the measurements from the volume flow meter that read total volume flow and the reading from the stop watch. Here the estimate volume flow rate $(\dot{V})$ is equal to the measured water volume $(V)$ divided by the measured time $(t)$.

$\dot{V}=\frac{V}{t}$

Following two equation have been derived by taking partial derivative of volume flow rate with respect to measured volume and with respect to measure time,

$$
\begin{aligned}
& \frac{\dot{\partial V}}{\partial V}=\frac{1}{t} \partial V \\
& \frac{\partial \dot{V}}{\partial t}=-\frac{V}{t^{2}} \partial t
\end{aligned}
$$

The following equation used to estimate the absolute uncertainty in the estimation of volume flow rate $(\Delta \dot{V})$ has been derived using the above two partial derivatives and the law of error propagation,

$$
\Delta \dot{V}=\sqrt{\left(\frac{1}{t} \Delta V\right)^{2}+\left(-\frac{V}{t^{2}} \Delta t\right)^{2}}
$$

The relative uncertainty in the estimation of volume flow rate is the ratio of the absolute uncertainty and the estimated volume flow rate as shown in the following equation. 


$$
\frac{\Delta \dot{V}}{\dot{V}}=\frac{\sqrt{\left(\frac{1}{t} \Delta V\right)^{2}+\left(-\frac{V}{t^{2}} \Delta t\right)^{2}}}{\dot{V}}
$$

Here, $(\Delta V)$ is the absolute uncertainty of the volume flow meter as specified by the flow meter manufacturer, $(\Delta t)$ is the absolute uncertainty of the stop watch as specified by the stop watch manufacturer including the human reflex uncertainty. The human reflex uncertainty becomes negligible for time measurements of above 30sec.

The relative velocity $V_{r}=\frac{\dot{V}}{A}$ of water jets at both the exit nozzles is equal to the estimated volume flow rate $(\dot{V})$ divided by the total exit nozzle area $(A)$. So the absolute uncertainty in the estimation of the velocity can be calculated using the following equation derived using the law of error propagation,

$$
\Delta V_{r}=\sqrt{\left(\frac{1}{A} \Delta \dot{V}\right)^{2}+\left(-\frac{\dot{V}}{A^{2}} \Delta A\right)^{2}}
$$

$$
\frac{\Delta V_{r}}{V_{r}}=\frac{\sqrt{\left(\frac{1}{A} \Delta \dot{V}\right)^{2}+\left(-\frac{\dot{V}}{A^{2}} \Delta A\right)^{2}}}{V_{r}}
$$

The absolute uncertainty in estimating the value of $k$-factor can be calculated from the following equation. This equation has been derived from the law of propagation and by 
using the value of estimated relative velocity, measured rotational speed, measured head and equation (37).

$$
\Delta k=\sqrt{\left(\frac{2 g \times \Delta H}{V_{r}^{2}}\right)^{2}+\left(\frac{2 R \omega^{2} \Delta R}{V_{r}^{2}}\right)^{2}+\left(\frac{2 \omega R^{2} \Delta \omega}{V_{r}^{2}}\right)^{2}+\left(\frac{-1}{\left(V_{r}^{2}\right)^{2}}\left(2 g H+R^{2} \omega^{2}\right) \times \Delta V_{r}\right)}
$$

Following the similar procedure as discussed above uncertainty analysis of the experimental performance results has been conducted. The uncertainty analysis of the experimental data from stationary test shows that the estimated torque has a relative uncertainty of $\pm 4.41 \%$ and the measured torque has a relative uncertainty of $\pm 1.13 \%$. The relative uncertainty in the measured volume flow rate is about $\pm 2.5 \%$ and the relative uncertainty in the estimated $k$-factor is only $\pm 2.53 \%$. The relative uncertainty in the estimation of the shaft power is estimated as maximum $\pm 5.02 \%$. The relative uncertainty in the estimation of the rate of potential energy supplied to the turbine is estimated as $\pm 2.70 \%$. From these calculated quantities of the relative uncertainties it is confirmed that the instrumentation and experimental procedures used for conducting the performance tests on the simple reaction turbine models have very high level of confidence and reliability.

\section{Conclusion}

The performance of an idealized reaction turbine has been represented in dimensionless equations and curves. For the ideal situation of a flow system and turbine without fluid friction losses, it is shown that the self pumping action implicit in energy conservation modifies the traditional expectations for this class of reactive turbine. In particular, the speed, power and mass flow rate will tend to infinities as the torque approaches half of 
the stalled value. From the theoretical analysis it is seen that mass flow rate increases with the increase in the rotational speed due to the centrifugal pumping action. The variation of $\dot{m}$ with rotational speed is almost linear and the effect of supply head on the mass flow rate vanishes at high rotational speeds. The self-pumping effect, i.e. increase in mass flow rate with rotational speed is true for the practical case with frictional losses and experimental results have validated this phenomenon.

The ideal characteristics modeled suggest that the simple reaction turbine may have application to high speed power generation from low head water supplies. As shown by the non-dimensional analysis a simple reaction water turbine has higher efficiency at high speeds. Although it is seen that for a practical situation with fluid frictional losses the simple reaction turbine will never experience the runaway condition of infinite speed. Further the maximum power and efficiency of a turbine with frictional losses will dependent on the $k$-factor and this has been concluded from the experimental results.

The test results have shown that turbine model 2 has higher turbine efficiency as compared with turbine model 1 for the same supply head, but at higher rotational speeds. This behavior of the turbine model 2 can be attributed to the fact that the model 2 has smaller diameter and hence the wet surface area is less. This helps to reduce the energy loss from the internal water drag that is prominent in the turbine model 1 due to larger diameter and hence larger wet surface area.

The uncertainty analysis has shown that the experimentally measured results and the estimated results have reasonable relative uncertainties and so the findings of this study can be used as a basis for future investigation. This analysis shows that a simple reaction turbine can convert low head hydro power to mechanical power with high efficiency. It is 
also very simple to fabricate the simple reaction turbines that have been experimentally investigated.

\section{References}

1. Wilson, P.N., Water Turbines. A Science Museum Booklet. 1974: Her Majesty's Stationery Office.

2. Balje, O.E., "Turbo-machines, a guide to design, selection and theory". 1981: John Wiley \& Sons.

3. Bartle, A., Hydropower potential and development activities. Energy Policy, 2002. 30(14): p. 1231-1239.

4. Shepherd, D.G., Principles of turbomachinery. 1956: Macmillan.

5. Leo, B.S. and S.T. Hsu, A simple reaction turbine as a solar engine. Solar Energy, 1960. 4(2): p. 16-20.

6. J.F., B., "The Rotating Nozzle (Hero's) Turbine". ASME, 1960(60-WA-293).

7. Daugherty, R.L., Franzini, Joseph B. Finnemore, E. John., Fluid mechanics with engineering applications. 1989: McGraw-Hill.

8. Balje, O.E., Turbomachines: A guide to design, selection and theory. 1981, NY: John Wiley.

9. Duncan W.J., T.A.S., and Young A.D., Mechanics of Fluids. 1970: Edward Arnold.

10. Davis, J.R.A., Science in Modem Life : Engineering. 1st ed. Science in Modem Life, ed. F. J.W. Vol. 6. 1910, London UK: The Gresham Publishing Company.

11. Daugherty R.L., I.A.G., Fluid Mechanics with Engineering Applications. 1954: McGraw-Hill Book Company. 
12. R.K., T., Principles of Turbomachinery. 1984: E. \& F.N. Spon.

13. Date, A., Low head simple reaction water turbine, in School of Aerospace Mechanical and Manufacturing Engineering2009, RMIT University: Melbourne. p. 251.

14. Date, A. and A. Akbarzadeh, Design and analysis of a split reaction water turbine. Renewable Energy, 2010. 35(9): p. 1947-1955.

15. Drosg, M., Dealing with Uncertainties A Guide to Error Analysis. 2007, Berlin: Springer-Verlag 